\title{
Spectral optical monitoring of the double-peaked emission line AGN Arp 102B
}

\section{Variability of the broad line properties ${ }^{\star}$}

\author{
L. Č. Popovićí1,2, A. I. Shapovalova ${ }^{3}$, D. Ilić ${ }^{2,4}$, A. N. Burenkov ${ }^{3}$, V. H. Chavushyan ${ }^{5}$, W. Kollatschny ${ }^{6}$, \\ A. Kovačević ${ }^{2,4}$, J. R. Valdés ${ }^{5}$, J. León-Tavares ${ }^{5,8}$, N. G. Bochkarev ${ }^{7}$, V. Patiño-Álvarez ${ }^{5}$, and J. Torrealba ${ }^{5}$ \\ 1 Astronomical Observatory, Volgina 7, 11160 Belgrade 74, Serbia \\ e-mail: ashap@sao.ru \\ 2 Isaac Newton Institute of Chile, Yugoslavia Branch, 11060 Belgrade, Serbia \\ 3 Special Astrophysical Observatory of the Russian AS, Nizhnij Arkhyz, 369167 Karachaevo-Cherkesia, Russia \\ 4 Department of Astronomy, Faculty of Mathematics, University of Belgrade, Studentski trg 16, 11000 Belgrade, Serbia \\ 5 Instituto Nacional de Astrofísica, Óptica y Electrónica, Apartado Postal 51, CP 72000, Puebla, Pue. México, Mexico \\ ${ }^{6}$ Institut für Astrophysik, Friedrich-Hund-Platz 1, Göttingen, Germany \\ 7 Sternberg Astronomical Institute, Moscow, Russia \\ ${ }^{8}$ Finnish Centre for Astronomy with ESO (FINCA), University of Turku, Väisäläntie 20, 21500 Piikkiö, Finland
}

Received 1 February 2014 / Accepted 23 August 2014

\section{ABSTRACT}

\begin{abstract}
Context. We investigate the long-term (26 years, from 1987 to 2013) variability in the broad spectral line properties of the radio galaxy Arp 102B, an active galaxy with broad double-peaked emission lines. We use observations presented in Paper I in the period from 1987 to 2011, and a new set of observations performed in 2012-2013.

Aims. We explore variations in the $\mathrm{H} \alpha$ and $\mathrm{H} \beta$ line parameters during the monitored period to explore the broad-line region (BLR) geometry and to clarify some contradictions about the nature of the BLR in Arp102B.

Methods. We fit the broad lines with three broad Gaussian functions finding the positions and intensities of the blue and red peaks in $\mathrm{H} \alpha$ and $\mathrm{H} \beta$. Additionally, we fit averaged line profiles with the disk model.

Results. We find that the broad line profiles are double peaked and have not been changed significantly in shapes, beside an additional small peak that from time to time can be seen in the blue part of the $\mathrm{H} \alpha$ line. The positions of the blue and red peaks have not changed significantly during the monitored period. The $\mathrm{H} \beta$ line is broader than $\mathrm{H} \alpha$ line in the monitored period. The disk model is able to reproduce the $\mathrm{H} \beta$ and $\mathrm{H} \alpha$ broad line profiles, however, observed variability in the line parameters are not in good agreement with the emission disk hypothesis.

Conclusions. It seems that the BLR of Arp 102B has a disk-like geometry, but the role of an outflow can also play an important role in observed variation of the broad line properties.
\end{abstract}

Key words. galaxies: active - quasars: individual: Arp 102B - line: profiles

\section{Introduction}

Arp 102B, a low-ionization nuclear emission-line region (LINER) like object, was the first active galactic nuclei (AGN) where the broad line double-peaked profiles have been modeled with emission of an accretion disk (Chen et al. 1989; Chen \& Halpern 1989). After that, this galaxy has been widely accepted as a prototype of an AGN with broad lines emitted from the disk (see Chen \& Halpern 1989; Sulentic et al. 1990; Eracleous \& Halpern 1994; Eracleous et al. 1997) and has been studied intensively through different monitoring campaigns (see, e.g., Miller \& Peterson 1990; Newman et al. 1997; Sergeev et al. 2000; Gezari et al. 2007; Shapovalova et al. 2013).

In general, the monitoring campaigns agree that the broadline region (BLR) in Arp 102B, where the double-peaked broad emission lines are forming, seems to have a disk-like

\footnotetext{
* Appendix A is available in electronic from at http://www. aanda.org
}

geometry, but there are still some open issues and contradictions concerning the disk model (Miller \& Peterson 1990; Newman et al. 1997; Sergeev et al. 2000; Gezari et al. 2007; Shapovalova et al. 2013). The broad $\mathrm{H} \alpha$ line profile variation, observed by Sergeev et al. (2000) from 1992 to 1996, corresponds to gas rotating in the disk with inhomogeneity in the surface brightness, which is in agreement with Gezari et al. (2007), who found that the accretion disk is the most favorable model. However, Gezari et al. (2007) concluded that most of the observed facts fail to explain the variability of the profiles assuming processes in the accretion disk. Additional disagreement with the disk model was reported by Miller \& Peterson (1990), who showed that, at least at some epochs, the long-wavelength side of the line profile is higher than the short-wavelength side. Miller \& Peterson's finding is contrary to what is expected from a relativistic disk, i.e., an asymmetric relativistic disk cannot explain the peak ratio observed in some periods. A sinusoidal variation of the red-to-blue peak flux ratio is present in the $\mathrm{H} \alpha$ line profile (Newman et al. 1997; Shapovalova et al. 2013). This variation 
can be explained as a transient orbiting hot spot in the accretion disk $^{1}$.

Additionally, it is confirmed that high ionized lines, such as $\operatorname{Ly} \alpha$ and CIV $\lambda 1550$, do not show the disk-like profile (two peaks) and that the $\operatorname{Ly} \alpha$ line is single peaked (Halpern et al. 1996), i.e., that the $\operatorname{Ly} \alpha / \mathrm{H} \beta$ ratio is less than 0.12 in the displaced peaks. The lack of double-peaked high ionization lines from the disk can indicate that the disk medium is too dense for those lines and that they cannot be emitted from the disk. However, it is interesting that in the near-infrared there is no trace of doublepeaked HI lines (Riffel et al. 2006). Moreover, both the Bracket and Paschen series are almost absent apart from $\mathrm{Pa} \alpha$ and $\mathrm{Pa} \beta$.

One way to confirm the disk emission in the broad lines is to observe polarization in broad lines (see, e.g., Corbett et al. 1998, 2000; Afanasiev et al. 2014), since the expected position angle of polarization should be parallel to the disk plane. This angle is the same as the direction of the radio jet (Antonucci et al. 1996; Corbett et al. 1998, 2000), which is not expected for the broad lines emitted from an accretion disk. Also, Chen et al. (1997) found discrepancies between the disk model and the spectropolarimetric data, but they found that if some specific conditions are taken into account, the observations could be explained with emission of the accretion disk.

Although the double-peaked broad lines of Arp 102B indicate an accretion disk emission, there are some deviations in broad line profile variations that may indicate another geometry. In Paper I (Shapovalova et al. 2013), we present observations and variation in line fluxes and the continuum. In this paper, we discuss the long-term spectral line profile variations in order to clarify the nature of the BLR in Arp 102B. The paper is organized as follows: in Sect. 2, we describe our observations and used methods of analysis; in Sect. 3, we present our results of the line profile variation analysis; in Sect. 4, we discuss our results; and finally, in Sect. 5, we outline our conclusions.

\section{Observations and methods of analysis}

\subsection{Observations}

Spectra of Arp 102B were taken during the monitoring period 1987-2010 with five different telescopes: two telescopes in Russia (the $6 \mathrm{~m} \mathrm{BTA}$ and $1 \mathrm{~m}$ Zeiss telescopes of Special Astrophysical Observatory (SAO), Russian Academy of Science), two $2.1 \mathrm{~m}$ telescopes in Mexico (Guillermo Haro Observatory, GHO, and Observatorio Astronomico Nacional at San Pedro Martir, SPM), and two telescopes in Spain (3.5 m and $2.2 \mathrm{~m}$ telescopes of Calar Alto Observatory, CA). Details of observations and data reductions are given in Paper I, and will not be repeated here.

In addition to the observational data presented in Paper I, we added ten new spectra observed in the 2012-2013 period with $2.1 \mathrm{~m}$ telescope of GHO. The measurements of the continuum at $5100 \AA$, $\mathrm{H} \alpha$, and $\mathrm{H} \beta$ fluxes are listed in Table 1. In Fig. 1, we plot the light curves in the continuum and in the broad lines to compare our new observations with those obtained in Paper I. As can be seen from Fig. 1, the minimum in the line flux is observed in 2012-2013, while the continuum flux is similar to that observed in the 1989-1992 period.

\footnotetext{
1 Similar variation can also be a consequence of gravitational lensing from a massive body close to a primary black hole (Popović et al. 2001).
}

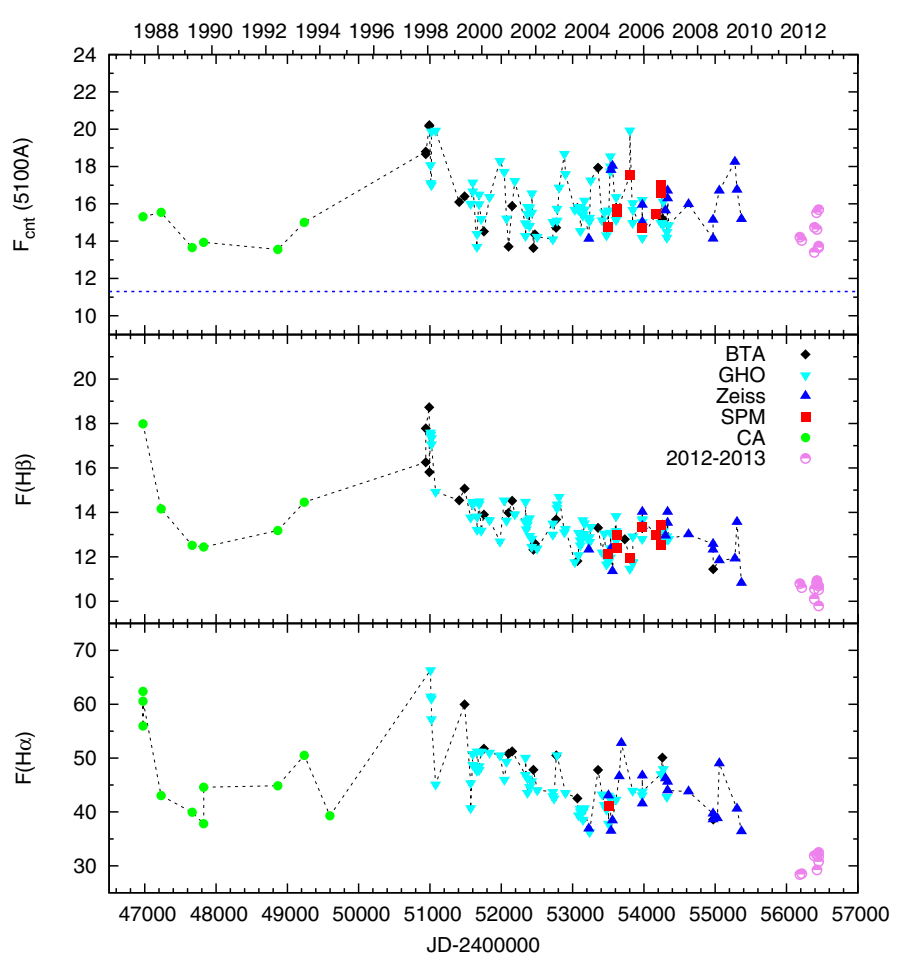

Fig. 1. Light curves with the new data in the 2012-2013 period. From top to bottom: blue continuum flux, $\mathrm{H} \beta$, and $\mathrm{H} \alpha$ line flux. Observations with different telescopes are denoted with different symbols given in the middle plot. The continuum flux is plotted in units of $10^{-16} \mathrm{erg} \mathrm{cm}^{-2} \mathrm{~s}^{-1} \AA^{-1}$, and the line flux in units of $10^{-14} \mathrm{erg} \mathrm{cm}^{-2} \mathrm{~s}^{-1}$. The dashed line in the blue and red continuum light curves mark the contribution of the host galaxy starlight continuum.

Table 1. Measurements of line fluxes of the new set of observations in the 2012-2013 period.

\begin{tabular}{lccccc}
\hline \hline$N$ & UT-date & MJD & $F_{\text {cnt }} 5100 \AA$ & $F(\mathrm{H} \beta)$ & $F(\mathrm{H} \alpha)$ \\
\hline 1 & 2012Sep.17 & 56187.69 & 14.21 & 10.78 & 28.40 \\
2 & 2012Oct.14 & 56214.67 & 14.03 & 10.61 & 28.53 \\
3 & 2013Apr.07 & 56389.99 & 13.39 & 10.10 & 31.88 \\
4 & 2013Apr.11 & 56393.98 & 14.74 & 10.55 & - \\
5 & 2013May 10 & 56422.87 & 15.52 & 10.82 & 32.07 \\
6 & 2013May 13 & 56425.82 & 14.63 & 10.94 & \\
7 & 2013May 14 & 56426.81 & - & - & 29.22 \\
8 & 2013Jun.06 & 56449.86 & 13.65 & 10.52 & 32.49 \\
9 & 2013Jun.07 & 56450.87 & 15.69 & 10.69 & 31.76 \\
10 & 2013Jun.08 & 56451.87 & 13.73 & 9.78 & 30.76 \\
\hline
\end{tabular}

Notes. The continuum flux is in units of $10^{-16} \mathrm{erg} \mathrm{cm}^{-2} \mathrm{~s}^{-1} \AA^{-1}$, and the line flux in units of $10^{-14} \mathrm{erg} \mathrm{cm}^{-2} \mathrm{~s}^{-1}$.

\subsection{Method of analysis}

To explore the broad line profile variability in detail, we performed the following method of analysis:

a) To subtract the narrow lines and obtain only the broad profiles, we fitted all $\mathrm{H} \alpha$ and $\mathrm{H} \beta$ lines with Gaussian functions. There is a problem with removing the narrow lines in Arp 102B, since they are right on top of the red peak in both broad lines $(\mathrm{H} \alpha$ and $\mathrm{H} \beta)$ and the measured properties of the red peak can be strongly affected by the narrow line removal procedure. We discuss this in more detail 

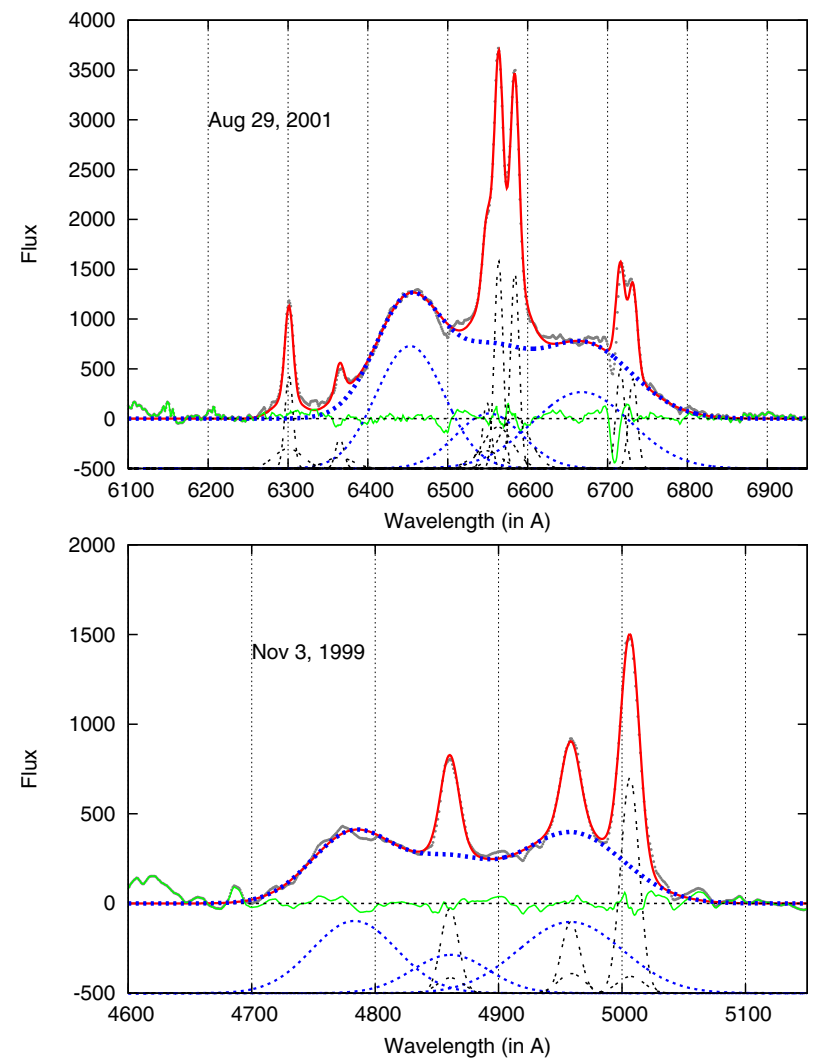

Fig. 2. Examples of the best Gaussian fit of one epoch $\mathrm{H} \alpha$ (upper panel) and $\mathrm{H} \beta$ line (bottom panel). Below the observed spectrum (dots), model (solid line), and residual (thin solid line), the Gaussian components are given, where broad components are denoted with dashed line and narrow components with double dashed line. The thick dashed line reproduce the modeled broad component of the fitted Balmer line.

in the Appendix, and here we briefly describe the subtraction procedure. To estimate the narrow line contribution we fitted the entire $\mathrm{H} \alpha$ and $\mathrm{H} \beta$ profiles (broad + narrow lines) simultaneously. We used three broad Gaussian functions for the broad component of the $\mathrm{H} \alpha$ and $\mathrm{H} \beta$ lines (Fig. 2), while we assumed the same widths and shifts for all narrow lines (see Popović et al. 2004). We assumed that the ratio of [OIII]4959 and [OIII]5007 follows the flux ratio 1:3 (Dimitrijević et al. 2007), and made the same assumption for [NII] doublet: $F[\mathrm{NII}] 6584 / F[\mathrm{NII}] 6548 \sim 3$. In the $\mathrm{H} \alpha$ wavelength band, the [SII]6717,6731 doublet, and [OI]6300 and [OI]6363 lines were fitted using single Gaussian. Note here that only one Gaussian cannot properly fit the narrow line wings, therefore, we included an additional, broader, Gaussian (with significantly smaller intensity) to fit the narrow line wings. As can be seen in Fig. 2, the three broad components (red and blue shifted, and central component) can satisfactorily explain the broad line profiles. To check the narrow line subtraction we perform several tests, see Appendix A for details.

b) After subtraction of the narrow lines and continuum, we made month-averaged line profiles using similar profiles (same peak positions and total line flux differs up to $\sim 10 \%$ ). These month-averaged line profiles are given for both $\mathrm{H} \alpha$ and $\mathrm{H} \beta$ line in Figs. 3 and 4, and we continued to measure and analyze properties of these profiles.

c) To determine the peak positions in the $\mathrm{H} \alpha$ and $\mathrm{H} \beta$ broad line profiles, we performed another Gaussian analysis, this time on the month-averaged broad profile. The broad profile was fitted with three Gaussian functions, corresponding to the blue, central, and red component (Fig. 5). From the inspection of the parameters obtained from the Gaussian fittings, we note that the central component very often changes its position, which affects other parameters, especially the intensity of the red peak. Therefore, we also fitted the month-averaged broad line profiles using the constraint that the central component should not have a large shift velocity, i.e., setting the limits on the shift of the central component between $-1000 \mathrm{~km} \mathrm{~s}^{-1}$ and $600 \mathrm{~km} \mathrm{~s}^{-1}$ (see Figs. 5 and 6 , and the discussion in Appendix A). The obtained error-bars of the parameters from both fittings are often smaller than the difference between the parameters obtained from these two fitting procedures, therefore we accepted the averaged value of the two best-fittings for the line parameters. For their error-bars, however, we took the corresponding standard deviation, i.e., the discrepancy between the averaged parameters and those obtained from the fits. The averaged parameters, with their uncertainties, of the Gaussian fittings of the broad $\mathrm{H} \alpha$ and $\mathrm{H} \beta$ components are listed in Tables 2 and 3 .

d) The broad double-peaked line profiles are usually fitted with some sort of disk model (see, e.g., Eracleous \& Halpern 1994; Gezari et al. 2007; Newman et al. 1997; Popović et al. 2011 , etc.). Here we use a relativistic disk model given by Chen et al. (1989) and Chen \& Halpern (1989) to model the broad $\mathrm{H} \alpha$ profile. In Fig. 7, we present a disk fitting with our parameters and those given in Gezari et al. (2007). We will discuss this in more details in Sect. 3.2.

e) Finally, we measured the full width at half maximum (FWHM) and full width at quarter (1/4) maximum (FWQM). These measurements are also provided in Tables 2 and 3.

\section{The broad line profile variability}

In Paper I, we measured and analyzed variations in the continuum and line fluxes using total of 118 spectra covering the $\mathrm{H} \beta$ wavelength region, and 90 spectra covering the $\mathrm{H} \alpha$ line. We showed that the fluxes in the $\mathrm{H} \alpha$ and $\mathrm{H} \beta$ lines and in continuum did not strongly vary (around $20 \%$ in line and $\sim 30 \%$ in continuum), and that several flare-like events were observed during the monitored period (1987-2010). Considering spectra from new observations, it seems that there is a minimum in the line emission in the 2012-2013 period (see Fig. 1), and also Fig. 4 reveals that both lines are weak, moreover, $\mathrm{H} \beta$ is very weak with two weak peaks comparable to noise.

During the monitored period, the broad $\mathrm{H} \alpha$ and $\mathrm{H} \beta$ lines have double-peaked profiles (even in the minimum), but, as shown in Figs. 3 and 4, their line shapes and widths are different. Comparing the month-averaged spectra of the $\mathrm{H} \alpha$ and $\mathrm{H} \beta$ broad lines (see Figs. 3 and 4), one can note that there is a difference between the peak ratio of $\mathrm{H} \alpha$ and $\mathrm{H} \beta$ lines. The $\mathrm{H} \alpha$ line mostly has a stronger blue peak, while very often the $\mathrm{H} \beta$ line has almost the same intensities of the blue and red peak. On the other side, the $\mathrm{H} \beta \mathrm{FWHM}$ and FWQM $\left(F W H M_{\text {mean }}=16100 \pm\right.$ $\left.700 \mathrm{~km} \mathrm{~s}^{-1}, F W Q M_{\text {mean }}=17900 \pm 700 \mathrm{~km} \mathrm{~s}^{-1}\right)$ are significantly broader than in the $\mathrm{H} \alpha$ line $\left(F W H M_{\text {mean }}=14400 \pm 400 \mathrm{~km} \mathrm{~s}^{-1}\right.$, $\left.F W Q M_{\text {mean }}=16600 \pm 800 \mathrm{~km} \mathrm{~s}^{-1}\right)$. This contradicts the disk model predictions, i.e., if $\mathrm{H} \beta$ originates closer to the central black hole (as indicated by larger line widths), it is expected that it has a more pronounced blue peak than $\mathrm{H} \alpha$.

On average the line profiles of the $\mathrm{H} \alpha$ and $\mathrm{H} \beta$ lines do not change significantly besides some flare-like variations seen 

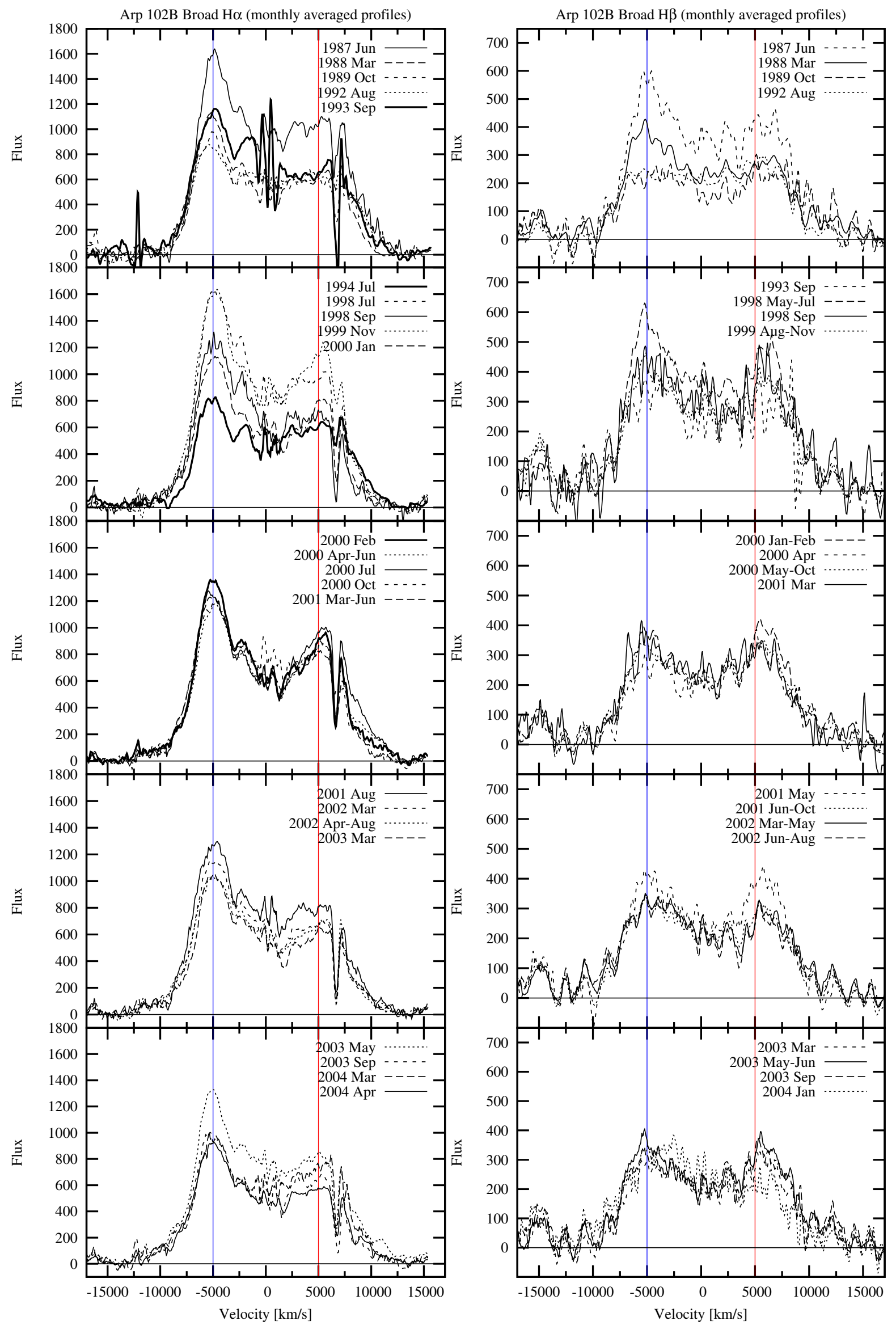

Fig. 3. Month-averaged profiles of the $\mathrm{H} \alpha$ and $\mathrm{H} \beta$ broad emission lines in the period between 1987-2004. The abscissa (OX) shows radial velocities with respect to the narrow component of the $\mathrm{H} \alpha$ or $\mathrm{H} \beta$ line. The ordinate (OY) shows the flux in units of $10^{-16} \mathrm{erg} \mathrm{cm}^{-2} \mathrm{~s}^{-1} \AA^{-1}$. 


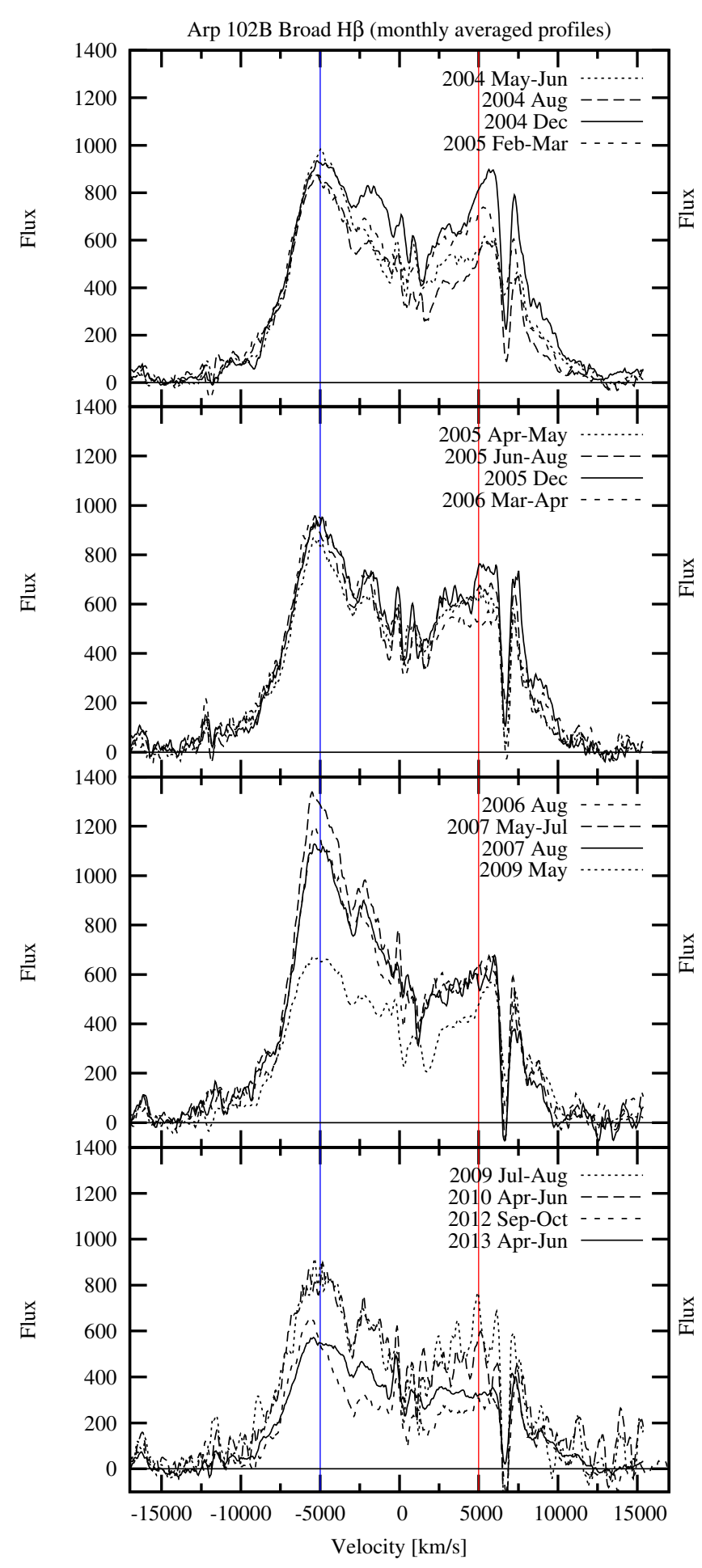

Fig. 4. Same as in Fig. 3, except for the period between 2004-2013.

in short periods (e.g., as in the period 1987-1992, 1998, and 2012-2013). In order to test the line profile changes, we normalized the blue peak of the month-averaged $\mathrm{H} \alpha$ profiles to unity and calculated the mean and root mean square (rms) profiles (Fig. 8). The normalized $\mathrm{H} \alpha$ rms profile shows that there is practically no change in the line profile. The change is below $10 \%$ in the red wing, where some variations may be due to uncertainty of the narrow line subtraction. Thus, considering the whole 26-year period, the averaged $\mathrm{H} \alpha$ line profile variation is practically neglected.

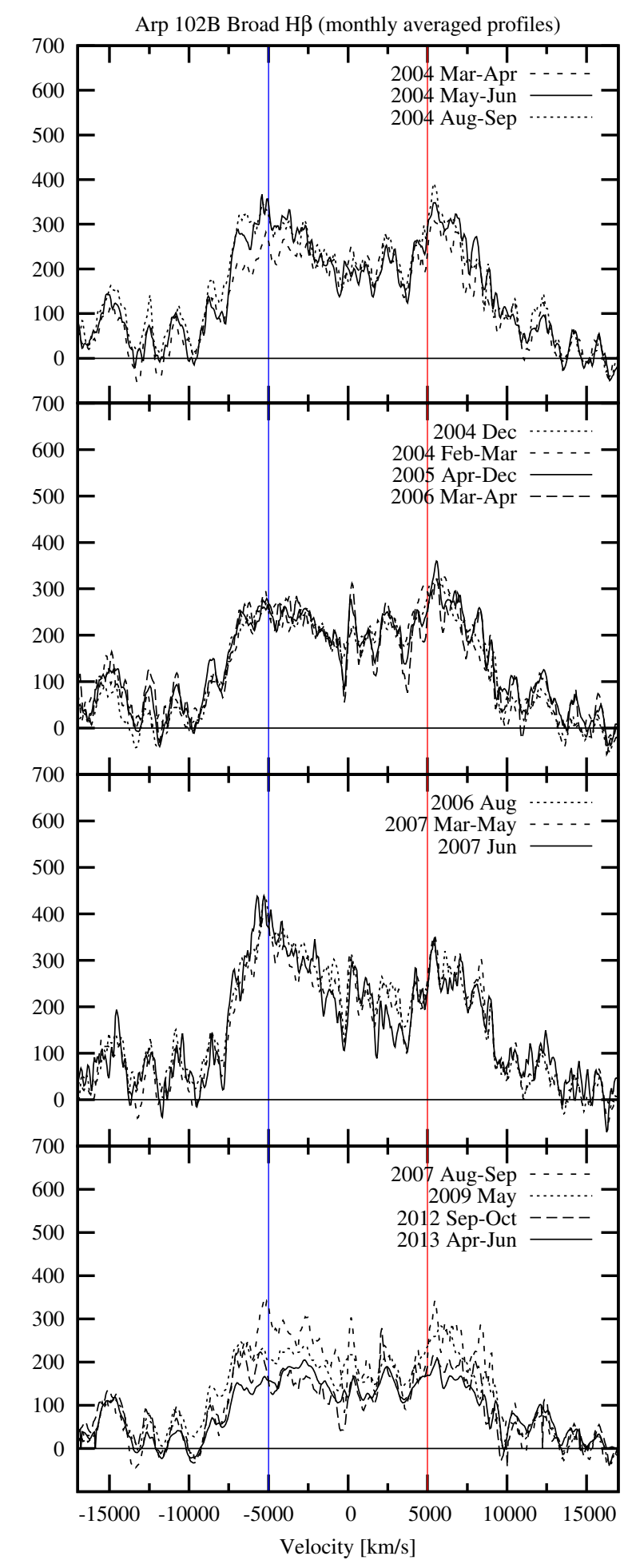

\subsection{Gaussian analysis: changes in the broad line profiles}

The parameters from the Gaussian fittings are listed in Tables 2 and 3. As shown from Tables 2 and 3 , the $\mathrm{H} \alpha$ and $\mathrm{H} \beta$ bluepeak positions change during the observed period for about $\pm 500 \mathrm{~km} \mathrm{~s}^{-1}$ around the averaged values (see Fig. 9 and Table 2), while the red-peak positions change for $\pm \sim 1000 \mathrm{~km} \mathrm{~s}^{-1}$ around averaged values, which may also be due to the measurement uncertainties. 
Table 2. Parameters of the Gaussian analysis of the broad $\mathrm{H} \alpha$ line profiles.

\begin{tabular}{|c|c|c|c|c|c|c|c|c|c|c|c|c|}
\hline \multirow[t]{2}{*}{ Year } & \multirow[t]{2}{*}{ Month } & \multicolumn{3}{|c|}{ 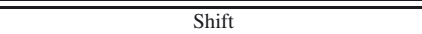 } & \multicolumn{3}{|c|}{ Intensity } & \multicolumn{3}{|c|}{ Width } & \multirow{2}{*}{$\begin{array}{c}F W H M \\
\mathrm{~km} \mathrm{~s}^{-1}\end{array}$} & \multirow{2}{*}{$\begin{array}{c}F W Q M \\
\mathrm{~km} \mathrm{~s}^{-1}\end{array}$} \\
\hline & & $\begin{array}{c}\text { blue } \\
\mathrm{km} \mathrm{s}^{-1}\end{array}$ & $\begin{array}{l}\text { central } \\
\mathrm{km} \mathrm{s}^{-1}\end{array}$ & $\begin{array}{c}\mathrm{red} \\
\mathrm{km} \mathrm{s}^{-1}\end{array}$ & blue & central & red & $\begin{array}{c}\text { blue } \\
\mathrm{km} \mathrm{s}^{-1}\end{array}$ & $\begin{array}{l}\text { central } \\
\mathrm{km} \mathrm{s}^{-1}\end{array}$ & $\begin{array}{c}\text { red } \\
\mathrm{km} \mathrm{s}^{-1}\end{array}$ & & \\
\hline 1987 & Jun. & $-5083 \pm 45$ & $-877 \pm 170$ & $5595 \pm 222$ & $1217 \pm 65$ & $866 \pm 66$ & $964 \pm 40$ & $2180 \pm 169$ & $4005 \pm 678$ & $3784 \pm 115$ & $14300 \pm 858$ & $16700 \pm 1002$ \\
\hline 1988 & Mar. & $-5250 \pm 131$ & $-638 \pm 512$ & $5720 \pm 106$ & $949 \pm 131$ & $576 \pm 21$ & $595 \pm 24$ & $2095 \pm 175$ & $3978 \pm 1132$ & $3224 \pm 74$ & $14500 \pm 870$ & $16100 \pm 966$ \\
\hline 1989 & Oct. & $-5400 \pm 4$ & $-793 \pm 293$ & $4983 \pm 447$ & $893 \pm 9$ & $424 \pm 58$ & $611 \pm 18$ & $2552 \pm 21$ & $2714 \pm 354$ & $4800 \pm 336$ & $15300 \pm 918$ & $18100 \pm 1086$ \\
\hline 1992 & Aug. & $-5562 \pm 140$ & $-475 \pm 289$ & $6392 \pm 319$ & $701 \pm 127$ & $607 \pm 18$ & $560 \pm 63$ & $2200 \pm 306$ & $4385 \pm 1216$ & $3617 \pm 185$ & $15800 \pm 948$ & $18100 \pm 1086$ \\
\hline 1993 & Sep. & $-5208 \pm 54$ & $-785 \pm 305$ & $5263 \pm 859$ & $1070 \pm 28$ & $710 \pm 72$ & $679 \pm 30$ & $2440 \pm 59$ & $2770 \pm 682$ & $4105 \pm 853$ & $15000 \pm 900$ & $17200 \pm 1032$ \\
\hline 1994 & Sep. & $-5241 \pm 91$ & $-721 \pm 395$ & $6019 \pm 182$ & $658 \pm 147$ & $487 \pm 12$ & $576 \pm 76$ & $1937 \pm 288$ & $4401 \pm 1603$ & $3641 \pm 79$ & $14000 \pm 840$ & $17000 \pm 1020$ \\
\hline 1998 & Jul. & $-4993 \pm 58$ & $-1239 \pm 338$ & $4847 \pm 642$ & $1317 \pm 230$ & $817 \pm 52$ & $876 \pm 120$ & $2246 \pm 44$ & $3847 \pm 1502$ & $3631 \pm 526$ & $14100 \pm 846$ & $15500 \pm 930$ \\
\hline 1998 & Sep. & $-4949 \pm 311$ & $-1381 \pm 539$ & $3925 \pm 49$ & $1165 \pm 92$ & $504 \pm 108$ & $675 \pm 49$ & $2594 \pm 243$ & $2116 \pm 581$ & $3925 \pm 161$ & $14300 \pm 858$ & $15300 \pm 918$ \\
\hline 1999 & Nov. & $-4981 \pm 194$ & $-453 \pm 770$ & $5281 \pm 337$ & $1363 \pm 336$ & $758 \pm 152$ & $995 \pm 78$ & $2344 \pm 366$ & $4163 \pm 1783$ & $2957 \pm 163$ & $14600 \pm 876$ & $15800 \pm 948$ \\
\hline 2000 & Jan. & $-5040 \pm 30$ & $-682 \pm 450$ & $5293 \pm 460$ & $896 \pm 233$ & $559 \pm 40$ & $632 \pm 167$ & $2163 \pm 413$ & $4805 \pm 2733$ & $2430 \pm 146$ & $13800 \pm 828$ & $15300 \pm 918$ \\
\hline 2000 & Feb. & $-5075 \pm 109$ & $-636 \pm 515$ & $5214 \pm 682$ & $1244 \pm 79$ & $592 \pm 40$ & $813 \pm 51$ & $2374 \pm 88$ & $3084 \pm 1273$ & $3293 \pm 646$ & $14100 \pm 846$ & $15600 \pm 936$ \\
\hline 2000 & Apr-Jun. & $-5001 \pm 188$ & $-842 \pm 56$ & $5059 \pm 491$ & $1061 \pm 99$ & $485 \pm 95$ & $787 \pm 35$ & $2598 \pm 109$ & $2820 \pm 1193$ & $3802 \pm 485$ & $14300 \pm 858$ & $16400 \pm 984$ \\
\hline 2000 & Jul. & $-5095 \pm 113$ & $-1037 \pm 52$ & $5193 \pm 267$ & $1198 \pm 46$ & $507 \pm 60$ & $944 \pm 39$ & $2413 \pm 80$ & $2437 \pm 543$ & $4013 \pm 350$ & $14600 \pm 876$ & $17400 \pm 1044$ \\
\hline 2000 & Oct. & $-5122 \pm 312$ & $-694 \pm 433$ & $5150 \pm 544$ & $930 \pm 337$ & $602 \pm 248$ & $675 \pm 205$ & $2406 \pm 630$ & $4316 \pm 2856$ & $3070 \pm 611$ & $14000 \pm 840$ & $16700 \pm 1002$ \\
\hline 2001 & Mar-Jun. & $-5237 \pm 74$ & $-884 \pm 165$ & $4929 \pm 333$ & $1147 \pm 33$ & $644 \pm 37$ & $776 \pm 10$ & $2558 \pm 43$ & $2817 \pm 556$ & $3299 \pm 354$ & $14200 \pm 852$ & $16500 \pm 990$ \\
\hline 2001 & Aug. & $-5135 \pm 119$ & $-957 \pm 59$ & $4459 \pm 490$ & $1214 \pm 47$ & $546 \pm 117$ & $788 \pm 21$ & $2613 \pm 75$ & $2514 \pm 633$ & $4254 \pm 445$ & $14300 \pm 858$ & $17000 \pm 1020$ \\
\hline 2002 & Mar. & $-5058 \pm 336$ & $-959 \pm 121$ & $4779 \pm 873$ & $1041 \pm 121$ & $511 \pm 192$ & $661 \pm 15$ & $2636 \pm 341$ & $2742 \pm 1405$ & $3684 \pm 784$ & $14200 \pm 852$ & $16100 \pm 966$ \\
\hline 2002 & Apr-Aug. & $-5027 \pm 288$ & $-666 \pm 172$ & $5008 \pm 465$ & $951 \pm 76$ & $466 \pm 116$ & $653 \pm 10$ & $2906 \pm 230$ & $2673 \pm 955$ & $3613 \pm 463$ & $14400 \pm 864$ & $16900 \pm 1014$ \\
\hline 2003 & Mar. & $-5097 \pm 238$ & $-1160 \pm 308$ & $5034 \pm 370$ & $978 \pm 55$ & $537 \pm 87$ & $592 \pm 5$ & $2311 \pm 258$ & $2496 \pm 689$ & $3831 \pm 420$ & $14000 \pm 840$ & $16100 \pm 966$ \\
\hline 2003 & May & $-5129 \pm 124$ & $-800 \pm 283$ & $4717 \pm 833$ & $1210 \pm 13$ & $564 \pm 193$ & $786 \pm 8$ & $2444 \pm 243$ & $2553 \pm 968$ & $4425 \pm 1043$ & $14100 \pm 846$ & $16800 \pm 1008$ \\
\hline 2003 & Sep. & $-5130 \pm 126$ & $-684 \pm 446$ & $4708 \pm 979$ & $930 \pm 40$ & $421 \pm 148$ & $640 \pm 16$ & $2534 \pm 365$ & $932 \pm 4016$ & $3374 \pm 820$ & $14300 \pm 858$ & $16600 \pm 996$ \\
\hline 2004 & Mar. & $-5185 \pm 66$ & $-865 \pm 192$ & $5568 \pm 292$ & $698 \pm 184$ & $533 \pm 67$ & $593 \pm 150$ & $2027 \pm 406$ & $5318 \pm 2233$ & $3494 \pm 363$ & $14600 \pm 876$ & $17600 \pm 1056$ \\
\hline 2004 & Apr. & $-5085 \pm 132$ & $-816 \pm 251$ & $4818 \pm 824$ & $857 \pm 26$ & $349 \pm 123$ & $542 \pm 40$ & $2551 \pm 343$ & $2851 \pm 1284$ & $4043 \pm 652$ & $14400 \pm 864$ & $16500 \pm 990$ \\
\hline 2004 & May-Jun. & $-5015 \pm 232$ & $-774 \pm 464$ & $5143 \pm 315$ & $860 \pm 100$ & $374 \pm 80$ & $538 \pm 33$ & $2637 \pm 206$ & $3181 \pm 1081$ & $3970 \pm 217$ & $14100 \pm 846$ & $16400 \pm 984$ \\
\hline 2004 & Aug. & $-5070 \pm 194$ & $-649 \pm 76$ & $5269 \pm 605$ & $811 \pm 41$ & $411 \pm 53$ & $528 \pm 17$ & $2798 \pm 208$ & $2553 \pm 1049$ & $3132 \pm 566$ & $14600 \pm 876$ & $16500 \pm 990$ \\
\hline 2004 & Dec. & $-4995 \pm 93$ & $-788 \pm 300$ & $5321 \pm 531$ & $860 \pm 61$ & $519 \pm 21$ & $775 \pm 6$ & $2837 \pm 12$ & $2717 \pm 1004$ & $3686 \pm 634$ & $14800 \pm 888$ & $18000 \pm 1080$ \\
\hline 2005 & Feb-Mar. & $-5150 \pm 124$ & $-631 \pm 522$ & $4893 \pm 482$ & $802 \pm 39$ & $375 \pm 42$ & $654 \pm 60$ & $3007 \pm 76$ & $2907 \pm 1185$ & $3490 \pm 501$ & $14600 \pm 876$ & $16900 \pm 1014$ \\
\hline 2005 & Apr. & $-5151 \pm 219$ & $-774 \pm 246$ & $4757 \pm 692$ & $797 \pm 4$ & $395 \pm 121$ & $611 \pm 15$ & $2552 \pm 641$ & $2664 \pm 1108$ & $3482 \pm 685$ & $14500 \pm 870$ & $17000 \pm 1020$ \\
\hline 2005 & Jun-Aug. & $-5237 \pm 240$ & $-927 \pm 104$ & $4887 \pm 521$ & $838 \pm 34$ & $389 \pm 113$ & $645 \pm 19$ & $2707 \pm 423$ & $2738 \pm 1072$ & $3485 \pm 515$ & $14700 \pm 882$ & $16900 \pm 1014$ \\
\hline 2005 & Dec. & $-5128 \pm 270$ & $-1044 \pm 62$ & $4884 \pm 294$ & $855 \pm 53$ & $420 \pm 85$ & $690 \pm 5$ & $2804 \pm 162$ & $2369 \pm 762$ & $3990 \pm 317$ & $14800 \pm 888$ & $17800 \pm 1068$ \\
\hline 2006 & Mar-Apr. & $-5065 \pm 257$ & $-800 \pm 283$ & $4710 \pm 759$ & $923 \pm 41$ & $351 \pm 210$ & $524 \pm 13$ & $2826 \pm 609$ & $2078 \pm 878$ & $4129 \pm 751$ & $14500 \pm 870$ & $17000 \pm 1020$ \\
\hline 2006 & Aug. & $-5039 \pm 273$ & $-1106 \pm 149$ & $4655 \pm 752$ & $1056 \pm 90$ & $496 \pm 156$ & $594 \pm 17$ & $2442 \pm 283$ & $2585 \pm 1007$ & $3684 \pm 584$ & $13700 \pm 822$ & $15200 \pm 912$ \\
\hline 2007 & May-Jul. & $-5164 \pm 391$ & $-1414 \pm 585$ & $4688 \pm 587$ & $1133 \pm 172$ & $652 \pm 172$ & $605 \pm 2$ & $2389 \pm 402$ & $2583 \pm 1024$ & $3590 \pm 397$ & $13400 \pm 804$ & $15300 \pm 918$ \\
\hline 2007 & Aug. & $-5163 \pm 327$ & $-1202 \pm 286$ & $4643 \pm 646$ & $1019 \pm 67$ & $624 \pm 146$ & $596 \pm 8$ & $2405 \pm 437$ & $2497 \pm 704$ & $3256 \pm 370$ & $13900 \pm 834$ & $15700 \pm 942$ \\
\hline 2009 & May & $-5265 \pm 223$ & $-981 \pm 21$ & $5420 \pm 401$ & $629 \pm 55$ & $373 \pm 33$ & $490 \pm 15$ & $2718 \pm 117$ & $2632 \pm 933$ & $3411 \pm 589$ & $14900 \pm 894$ & $17700 \pm 1062$ \\
\hline 2009 & Jul-Aug. & $-5249 \pm 528$ & $-1326 \pm 461$ & $4753 \pm 511$ & $769 \pm 65$ & $376 \pm 170$ & $568 \pm 11$ & $2927 \pm 616$ & $2167 \pm 1179$ & $3654 \pm 314$ & $14900 \pm 894$ & $16400 \pm 984$ \\
\hline 2010 & Apr-Jun. & $-5363 \pm 211$ & $-1190 \pm 268$ & $3724 \pm 1983$ & $704 \pm 63$ & $335 \pm 255$ & $478 \pm 62$ & $2602 \pm 165$ & $2106 \pm 1256$ & $5717 \pm 3209$ & $14700 \pm 882$ & $16700 \pm 1002$ \\
\hline 2012 & Sep-Oct. & $-5581 \pm 135$ & $-1173 \pm 244$ & $5274 \pm 477$ & $612 \pm 4$ & $274 \pm 40$ & $283 \pm 24$ & $1981 \pm 249$ & $2384 \pm 415$ & $3893 \pm 630$ & $14700 \pm 882$ & $16500 \pm 990$ \\
\hline 2013 & Apr-Jun. & $-5056 \pm 86$ & $-792 \pm 295$ & $4676 \pm 993$ & $537 \pm 6$ & $241 \pm 91$ & $333 \pm 4$ & $2823 \pm 66$ & $2365 \pm 675$ & $4499 \pm 877$ & $14800 \pm 888$ & $16800 \pm 1008$ \\
\hline mean & & $-5148 \pm 143$ & $-887 \pm 240$ & $5016 \pm 486$ & $946 \pm 208$ & $504 \pm 141$ & $649 \pm 150$ & $2502 \pm 269$ & $2955 \pm 880$ & $3728 \pm 553$ & $14431 \pm 448$ & $16618 \pm 790$ \\
\hline
\end{tabular}

Notes. Columns: shifts, intensities (given in $10^{-16} \mathrm{erg} \mathrm{cm}^{-2} \mathrm{~s}^{-1} \AA^{-1}$ ), width of the blue, central, and red Gaussian. Measurements of the FWHM and FWQM are also listed.

In general, the $\mathrm{H} \alpha$ profiles are of much better quality than $\mathrm{H} \beta$, thus we plotted in Fig. 10 the results of the peak-measurements of the $\mathrm{H} \alpha$ line: the blue- vs. red-peak intensity (upper panel), and the blue- vs. red-peak velocity (bottom panel). It is interesting that there is no correlation between the velocities of the red and blue peaks (see Fig. 10, bottom panel): there may be a trend toward an anticorrelation, which is statistically not significant $\left(r=-0.28, P_{0}=0.08\right)$, and it seems that they vary independently of each other. There is a good correlation, however, between the two peak intensities (see Fig. 10, upper panel), and the blue- and red-peak intensities are responding to each other.

In order to compare the position of the blue peak from our monitoring campaign with previous campaigns, we plot in Fig. 11 (upper panel) our measurements of the blue-peak velocity for the $\mathrm{H} \alpha$ line together with the measurements of Newman et al. (1997) and Gezari et al. (2007). As shown in Fig. 11, the agreement between different measurements is good, and the differences are within the error-bars $\left( \pm 100-200 \mathrm{~km} \mathrm{~s}^{-1}\right)$. In Fig. 11 (bottom panel), we compared the blue- and red-peak positions between $\mathrm{H} \alpha$ and $\mathrm{H} \beta$ during monitored period. As shown in Fig. 11 (bottom panel), there is a discrepancy between the positions of the red peaks of $\mathrm{H} \alpha$ and $\mathrm{H} \beta$ (in some periods for about $2000 \mathrm{~km} \mathrm{~s}^{-1}$ ), while the blue-peak position in both lines has no big change. There are small differences between the $\mathrm{H} \alpha$ and $\mathrm{H} \beta$ blue-peak positions, which are below $\sim 500 \mathrm{~km} \mathrm{~s}^{-1}$ (see also Tables 2 and 3 ).

In Fig. 12, we plot the $\mathrm{H} \alpha$ vs. $\mathrm{H} \beta$ peak velocity for the central (upper), blue (middle), and red (bottom) peak of Gaussians from the month-averaged profile fits. As shown in Fig. 12, there are no expected correlations, however, it seems that a trend of anticorrelation is present in the red $\mathrm{H} \alpha$ vs. $\mathrm{H} \beta$ velocities (solid line on the plot), while a correlation trend may be present between the blue-peak velocities of $\mathrm{H} \alpha$ and $\mathrm{H} \beta$. We should note that a trend of anticorrelation between the red-peak position may be caused by the measurement uncertainties, but that is not the case for the blue-peak positions. A theoretical expectation is that the velocity of the blue peak of the $\mathrm{H} \alpha$ line follows that in the $\mathrm{H} \beta$ line, and, as shown in Fig. 12 (middle) there may be a slight dependence between the blue-peak velocities of $\mathrm{H} \alpha$ and $\mathrm{H} \beta$, but it seems to be very weak (even taking the uncertainties in the measurements shown in the plots into account). This confirms that there are no expected correlations between the peak velocities of two broad lines.

\subsection{Disk model of the broad emission lines}

As we noted above, the relativistic disk model (Chen et al. 1989; Chen \& Halpern 1989; Gezari et al. 2007) can explain the broad double-peaked profiles of Arp 102B. We performed several fits, and it was interesting that the month-averaged profiles 
L. Č. Popović et al.: Spectral monitoring of Arp 102B. II.

Table 3. Same as in Table 2, except for the broad $\mathrm{H} \beta$ line.

\begin{tabular}{|c|c|c|c|c|c|c|c|c|c|c|c|c|}
\hline \multirow[t]{3}{*}{ Year } & \multirow[t]{3}{*}{ Month } & \multicolumn{3}{|c|}{ 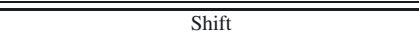 } & \multicolumn{3}{|c|}{ Intensity } & \multicolumn{3}{|c|}{ Width } & \multirow{3}{*}{$\begin{array}{c}F W H M \\
\mathrm{~km} \mathrm{~s}^{-1}\end{array}$} & \multirow{3}{*}{$\begin{array}{c}F W Q M \\
\mathrm{~km} \mathrm{~s}^{-1}\end{array}$} \\
\hline & & blue & central & red & blue & central & red & blue & central & red & & \\
\hline & & $\mathrm{km} \mathrm{s}^{-1}$ & $\mathrm{~km} \mathrm{~s}^{-1}$ & $\mathrm{~km} \mathrm{~s}^{-1}$ & & & & $\mathrm{~km} \mathrm{~s}^{-1}$ & $\mathrm{~km} \mathrm{~s}^{-1}$ & $\mathrm{~km} \mathrm{~s}^{-1}$ & & \\
\hline 1987 & Jun. & $-4930 \pm 326$ & $-440 \pm 791$ & $5747 \pm 434$ & $478 \pm 112$ & $257 \pm 76$ & $377 \pm 34$ & $2640 \pm 378$ & $3907 \pm 1692$ & $3889 \pm 360$ & $15300 \pm 918$ & $17500 \pm 1050$ \\
\hline 1988 & Mar. & $-5206 \pm 110$ & $-575 \pm 601$ & $6442 \pm 583$ & $320 \pm 109$ & $221 \pm 9$ & $235 \pm 63$ & $2445 \pm 282$ & $4831 \pm 2834$ & $3964 \pm 154$ & $15500 \pm 930$ & $17100 \pm 1026$ \\
\hline 1989 & Oct. & $-4974 \pm 47$ & $-515 \pm 687$ & $6035 \pm 54$ & $231 \pm 4$ & $111 \pm 10$ & $225 \pm 7$ & $3446 \pm 202$ & $2486 \pm 745$ & $4913 \pm 1017$ & $17800 \pm 1068$ & $18900 \pm 1134$ \\
\hline 1992 & Aug. & $-5714 \pm 496$ & $-847 \pm 216$ & $5263 \pm 189$ & $215 \pm 35$ & $157 \pm 27$ & $236 \pm 59$ & $2961 \pm 459$ & $3752 \pm 1299$ & $4050 \pm 21$ & $16100 \pm 966$ & $18800 \pm 1128$ \\
\hline 1993 & Sep. & $-4968 \pm 161$ & $378 \pm 315$ & $6499 \pm 1118$ & $279 \pm 119$ & $184 \pm 57$ & $223 \pm 89$ & $3248 \pm 78$ & $5688 \pm 4228$ & $3354 \pm 1184$ & $17100 \pm 1026$ & $18900 \pm 1134$ \\
\hline 1998 & May-Jul. & $-5217 \pm 156$ & $-332 \pm 945$ & $6533 \pm 48$ & $444 \pm 107$ & $380 \pm 16$ & $416 \pm 27$ & $2184 \pm 447$ & $4848 \pm 1200$ & $2911 \pm 34$ & $15400 \pm 924$ & $17100 \pm 1026$ \\
\hline 1998 & Sep. & $-5053 \pm 454$ & $-470 \pm 738$ & $5969 \pm 15$ & $406 \pm 42$ & $273 \pm 25$ & $374 \pm 8$ & $2932 \pm 394$ & $2669 \pm 803$ & $4043 \pm 62$ & $16200 \pm 972$ & $18400 \pm 1104$ \\
\hline 1999 & Aug-Nov. & $-5052 \pm 456$ & $-419 \pm 809$ & $5998 \pm 27$ & $391 \pm 20$ & $269 \pm 32$ & $372 \pm 6$ & $3009 \pm 502$ & $2777 \pm 650$ & $4010 \pm 109$ & $15600 \pm 936$ & $17100 \pm 1026$ \\
\hline 2000 & Jan-Feb. & $-4955 \pm 226$ & $-355 \pm 913$ & $6172 \pm 233$ & $285 \pm 93$ & $194 \pm 33$ & $329 \pm 53$ & $2554 \pm 695$ & $5034 \pm 3103$ & $3250 \pm 326$ & $15400 \pm 924$ & $17600 \pm 1056$ \\
\hline 2000 & Apr. & $-4928 \pm 96$ & $-311 \pm 972$ & $5608 \pm 559$ & $264 \pm 5$ & $139 \pm 38$ & $294 \pm 3$ & $2827 \pm 59$ & $3012 \pm 518$ & $4150 \pm 782$ & $15800 \pm 948$ & $17900 \pm 1074$ \\
\hline 2000 & May-Oct. & $-5249 \pm 8$ & $265 \pm 474$ & $6161 \pm 180$ & $243 \pm 71$ & $206 \pm 27$ & $241 \pm 84$ & $2547 \pm 485$ & $6032 \pm 3201$ & $3206 \pm 770$ & $16100 \pm 966$ & $18800 \pm 1128$ \\
\hline 2001 & Mar. & $-5152 \pm 210$ & $-475 \pm 743$ & $5274 \pm 317$ & $332 \pm 1$ & $183 \pm 5$ & $320 \pm 7$ & $2963 \pm 266$ & $2753 \pm 303$ & $3889 \pm 332$ & $15700 \pm 942$ & $17400 \pm 1044$ \\
\hline 2001 & May & $-5018 \pm 162$ & $-343 \pm 928$ & $6086 \pm 124$ & $300 \pm 107$ & $248 \pm 24$ & $358 \pm 46$ & $2583 \pm 544$ & $4989 \pm 2205$ & $2742 \pm 168$ & $14900 \pm 894$ & $17900 \pm 1074$ \\
\hline 2001 & Jun-Oct. & $-5137 \pm 394$ & $-448 \pm 781$ & $6167 \pm 441$ & $239 \pm 98$ & $192 \pm 52$ & $243 \pm 45$ & $2811 \pm 743$ & $4792 \pm 2630$ & $3482 \pm 523$ & $15700 \pm 942$ & $18200 \pm 1092$ \\
\hline 2002 & Mar-May & $-4802 \pm 125$ & $-372 \pm 796$ & $6385 \pm 37$ & $276 \pm 39$ & $133 \pm 3$ & $263 \pm 5$ & $4032 \pm 161$ & $3875 \pm 829$ & $4064 \pm 95$ & $16100 \pm 966$ & $18400 \pm 1104$ \\
\hline 2002 & Jun-Aug. & $-4465 \pm 470$ & $-350 \pm 919$ & $6196 \pm 142$ & $314 \pm 8$ & $83 \pm 66$ & $260 \pm 14$ & $4506 \pm 861$ & $1921 \pm 1100$ & $3700 \pm 187$ & $16100 \pm 966$ & $18900 \pm 1134$ \\
\hline 2003 & Mar. & $-4987 \pm 486$ & $-438 \pm 795$ & $6071 \pm 45$ & $239 \pm 35$ & $176 \pm 40$ & $281 \pm 3$ & $2904 \pm 426$ & $3586 \pm 340$ & $3415 \pm 67$ & $15500 \pm 930$ & $17400 \pm 1044$ \\
\hline 2003 & May-Jun. & $-5163 \pm 253$ & $-469 \pm 752$ & $5980 \pm 178$ & $335 \pm 12$ & $174 \pm 15$ & $325 \pm 3$ & $2882 \pm 235$ & $3205 \pm 46$ & $3926 \pm 206$ & $15800 \pm 948$ & $17300 \pm 1038$ \\
\hline 2003 & Sep. & $-5101 \pm 1$ & $-539 \pm 653$ & $5656 \pm 489$ & $284 \pm 15$ & $118 \pm 21$ & $287 \pm 13$ & $3233 \pm 129$ & $3235 \pm 1161$ & $4846 \pm 486$ & $16400 \pm 984$ & $17900 \pm 1074$ \\
\hline 2004 & Jan. & $-4184 \pm 462$ & $304 \pm 412$ & $5807 \pm 145$ & $309 \pm 19$ & $120 \pm 75$ & $197 \pm 27$ & $4465 \pm 1227$ & $4100 \pm 1563$ & $3582 \pm 22$ & $15000 \pm 900$ & $18200 \pm 1092$ \\
\hline 2004 & Mar-Apr. & $-4713 \pm 418$ & $359 \pm 342$ & $5726 \pm 168$ & $243 \pm 8$ & $119 \pm 55$ & $254 \pm 7$ & $3925 \pm 468$ & $2491 \pm 713$ & $4019 \pm 600$ & $15800 \pm 948$ & $18600 \pm 1116$ \\
\hline 2004 & May-Jun. & $-4858 \pm 110$ & $-130 \pm 1230$ & $6447 \pm 158$ & $249 \pm 99$ & $155 \pm 39$ & $253 \pm 60$ & $3027 \pm 802$ & $6152 \pm 4465$ & $3212 \pm 507$ & $16300 \pm 978$ & $18700 \pm 1122$ \\
\hline 2004 & Aug-Sep. & $-5293 \pm 28$ & $-394 \pm 851$ & $5831 \pm 223$ & $304 \pm 20$ & $122 \pm 54$ & $300 \pm 19$ & $3762 \pm 410$ & $3256 \pm 366$ & $4010 \pm 834$ & $16400 \pm 984$ & $18200 \pm 1092$ \\
\hline 2004 & Dec. & $-5308 \pm 506$ & $-346 \pm 926$ & $6031 \pm 177$ & $214 \pm 66$ & $169 \pm 24$ & $263 \pm 24$ & $3107 \pm 379$ & $4154 \pm 1635$ & $3449 \pm 2$ & $16600 \pm 996$ & $17800 \pm 1068$ \\
\hline 2005 & Feb-Mar. & $-5032 \pm 301$ & $446 \pm 9$ & $5889 \pm 134$ & $193 \pm 96$ & $175 \pm 33$ & $227 \pm 75$ & $2926 \pm 509$ & $5799 \pm 3966$ & $2813 \pm 901$ & $15300 \pm 918$ & $18000 \pm 1080$ \\
\hline 2005 & Apr-Dec. & $-5084 \pm 270$ & $-516 \pm 685$ & $6247 \pm 151$ & $181 \pm 106$ & $169 \pm 13$ & $224 \pm 71$ & $2936 \pm 431$ & $6546 \pm 4992$ & $3427 \pm 593$ & $17200 \pm 1032$ & $18100 \pm 1086$ \\
\hline 2006 & Mar-Apr. & $-4921 \pm 15$ & $-172 \pm 1169$ & $6495 \pm 64$ & $190 \pm 106$ & $168 \pm 23$ & $200 \pm 67$ & $2956 \pm 908$ & $6612 \pm 5120$ & $3071 \pm 753$ & $16400 \pm 984$ & $17700 \pm 1062$ \\
\hline 2006 & Aug. & $-4630 \pm 134$ & $505 \pm 134$ & $6096 \pm 71$ & $356 \pm 12$ & $146 \pm 7$ & $277 \pm 0$ & $3893 \pm 2$ & $2665 \pm 453$ & $3691 \pm 150$ & $16400 \pm 984$ & $16700 \pm 1002$ \\
\hline 2007 & May & $-5096 \pm 98$ & $-501 \pm 705$ & $6632 \pm 412$ & $251 \pm 98$ & $212 \pm 26$ & $250 \pm 59$ & $2504 \pm 906$ & $5492 \pm 3532$ & $3000 \pm 629$ & $16100 \pm 966$ & $17100 \pm 1026$ \\
\hline 2007 & Jun. & $-5138 \pm 240$ & $-505 \pm 701$ & $6435 \pm 211$ & $373 \pm 14$ & $186 \pm 12$ & $253 \pm 2$ & $2757 \pm 251$ & $2841 \pm 232$ & $4325 \pm 240$ & $16300 \pm 978$ & $16800 \pm 1008$ \\
\hline 2007 & Aug-Sep. & $-4957 \pm 235$ & $-470 \pm 750$ & $6637 \pm 463$ & $216 \pm 73$ & $182 \pm 31$ & $241 \pm 27$ & $2790 \pm 587$ & $4959 \pm 2125$ & $3166 \pm 382$ & $16300 \pm 978$ & $16700 \pm 1002$ \\
\hline 2009 & May & $-4797 \pm 414$ & $417 \pm 260$ & $5938 \pm 40$ & $234 \pm 6$ & $88 \pm 47$ & $242 \pm 4$ & $4595 \pm 987$ & $2283 \pm 613$ & $3735 \pm 80$ & $17300 \pm 1038$ & $18200 \pm 1092$ \\
\hline 2012 & Sep-Oct. & $-5593 \pm 308$ & $264 \pm 475$ & $6039 \pm 437$ & $149 \pm 57$ & $104 \pm 37$ & $146 \pm 52$ & $2587 \pm 784$ & $6780 \pm 5142$ & $3194 \pm 1567$ & $16000 \pm 960$ & $17700 \pm 1062$ \\
\hline 2013 & Apr-Jun. & $-4424 \pm 683$ & $300 \pm 425$ & $5363 \pm 810$ & $156 \pm 6$ & $69 \pm 60$ & $157 \pm 25$ & $3441 \pm 37$ & $1350 \pm 1907$ & $4531 \pm 989$ & $16700 \pm 1002$ & $18000 \pm 1080$ \\
\hline mean & & $-5003 \pm 298$ & $-220 \pm 374$ & $6054 \pm 365$ & $279 \pm 78$ & $173 \pm 63$ & $269 \pm 62$ & $3129 \pm 616$ & $4085 \pm 1484$ & $3677 \pm 544$ & $16076 \pm 654$ & $17882 \pm 663$ \\
\hline
\end{tabular}
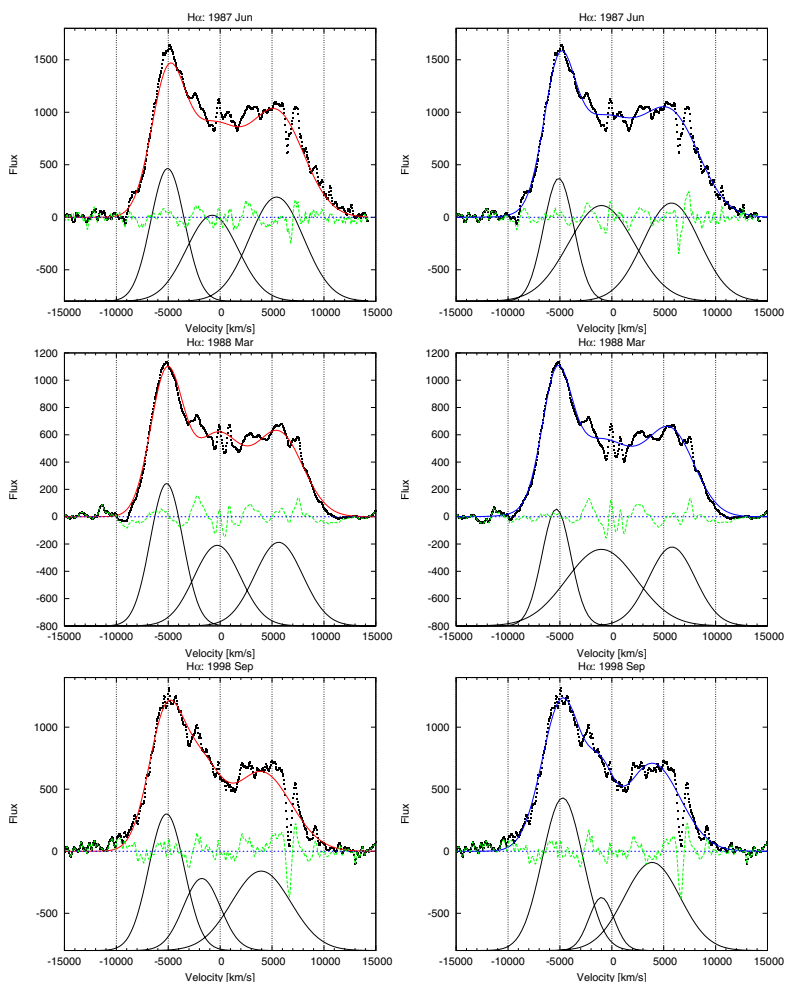

Fig. 5. Month-averaged $\mathrm{H} \alpha$ broad component fitted with Gaussians using two different assumptions: all parameters are free (left panels) and the shift of the central component is limited (right panels). Below the observed spectrum (dots), model (solid line), and residual (dashed line), the three broad Gaussian components are given. Each plot is labeled with the observed month and year.
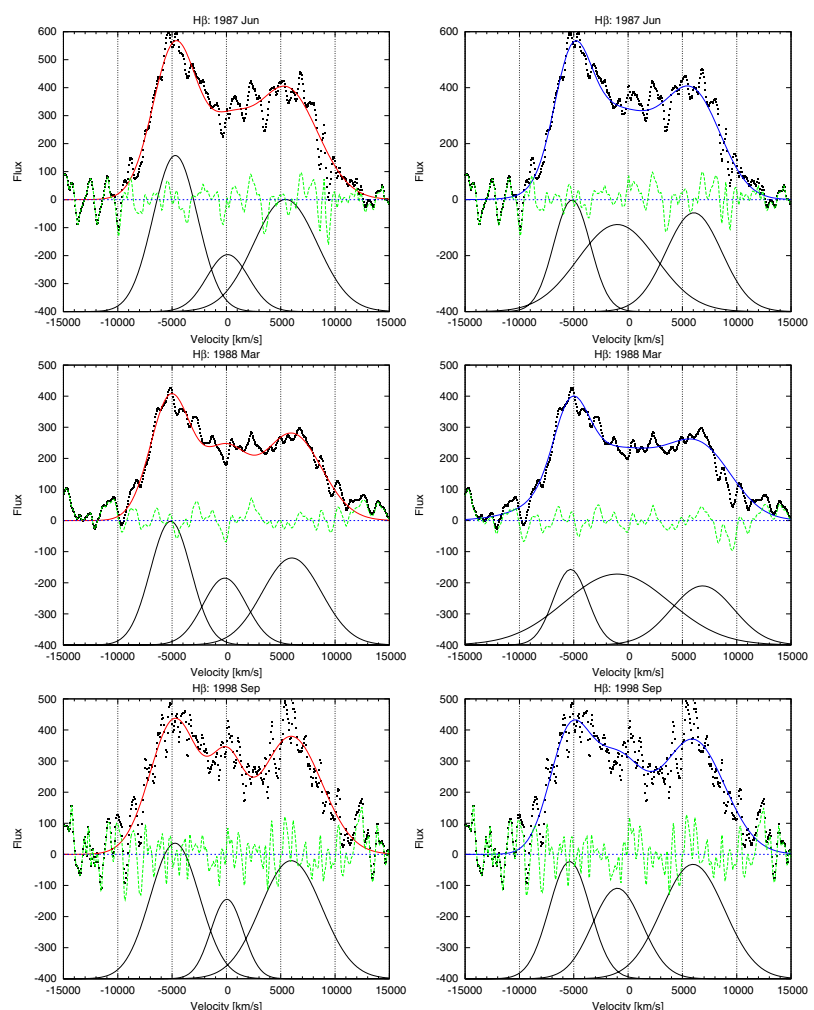

Fig. 6. Same as in Fig. 5, except for $\mathrm{H} \beta$ line.

can be successfully fitted with the simple Chen et al. (1989) disk model. The problem is that in some periods we could only fit 

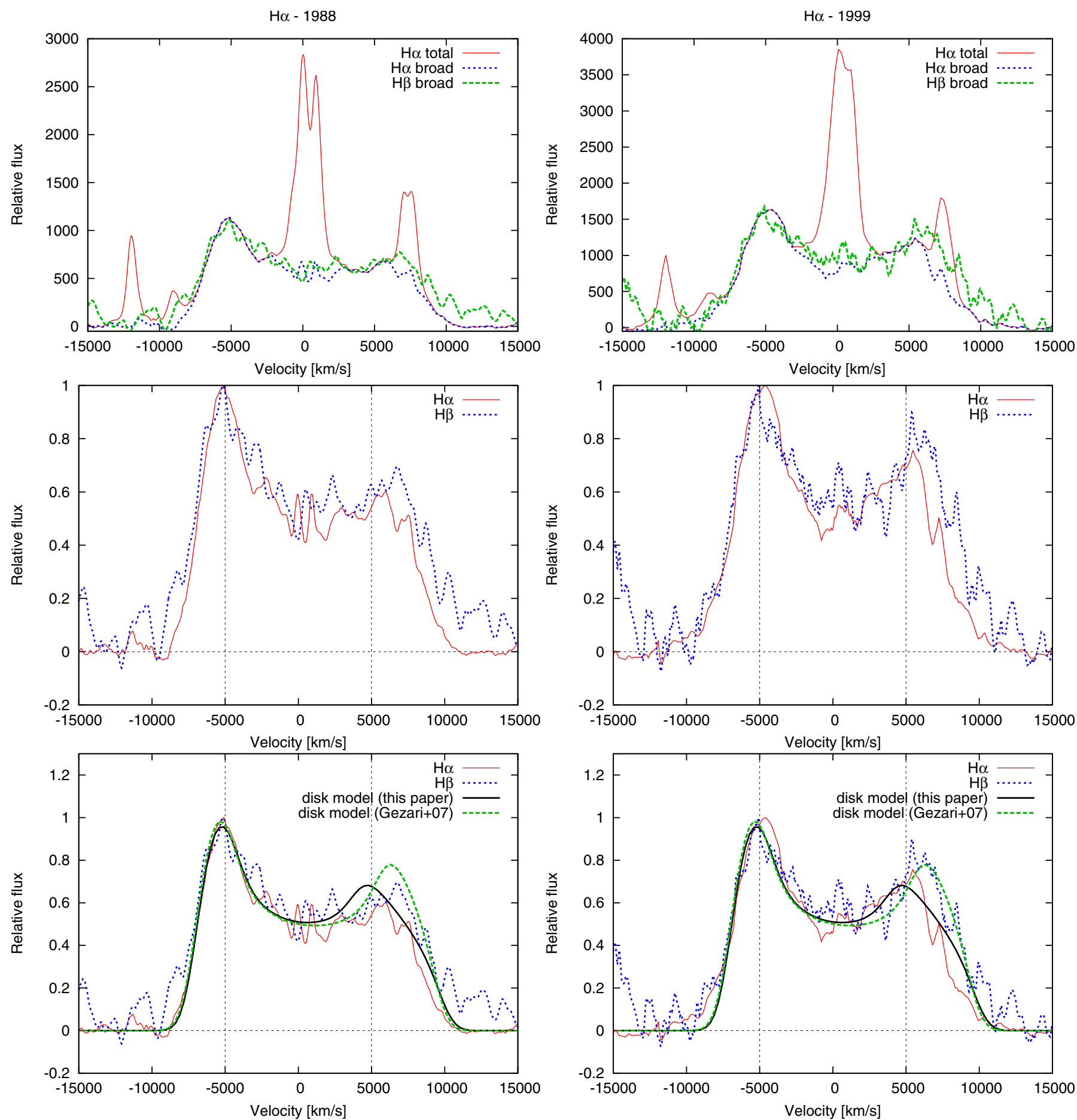

Fig. 7. $\mathrm{H} \alpha$ line profiles in 1988 (left panels) and 1999 (right panels). Upper panels: total $\mathrm{H} \alpha$ line (with narrow lines) compared with broad $\mathrm{H} \alpha$ and $\mathrm{H} \beta$ component (multiplied with the constant to match the $\mathrm{H} \alpha$ blue peak); middle panels: normalized broad $\mathrm{H} \alpha$ and $\mathrm{H} \beta$ component; bottom panels: disk models from this paper and Gezari et al. (2007).

the line profiles if we changed the inclination of the disk about 10 degrees, which is not expected. Note here that in the case when the inclination was fixed we obtained the satisfied fit by varying the other parameters (e.g., the emissivity and inner radius). We will not present all fits here, we only present examples of the fits with parameters given by Gezari et al. (2007): the inner radius $R_{\text {inn }} \sim 325 R_{\mathrm{g}}$, the outer radius $R_{\text {out }} \sim 825 R_{\mathrm{g}}$, the disk inclination $i \sim 31 \mathrm{deg}$, the random velocity in the disk $\sigma=1050 \mathrm{~km} \mathrm{~s}^{-1}$, with the emissivity $r^{-q}$, where $q=3.0$ is assumed (see Figs. 7 and 13). Additionally, starting from the parameters given above, we find the best fit of the mean $\mathrm{H} \alpha$ profile, by changing the inclination to $i=23 \mathrm{deg}$, and shifting the entire line for $-900 \mathrm{~km} \mathrm{~s}^{-1}$. As an example, these two fits are compared with observations from two periods for the $\mathrm{H} \alpha$ and $\mathrm{H} \beta$ line in Fig. 7 (bottom panels). The agreement between the observed blue peak and both models is good, but we see differences in the red wing (Fig. 7, bottom panels). We emphasize again, however, that the narrow line subtraction may contribute to the uncertainty in the red-peak properties, which can thus affect the obtained disk parameters. 
L. Č. Popović et al.: Spectral monitoring of Arp 102B. II.
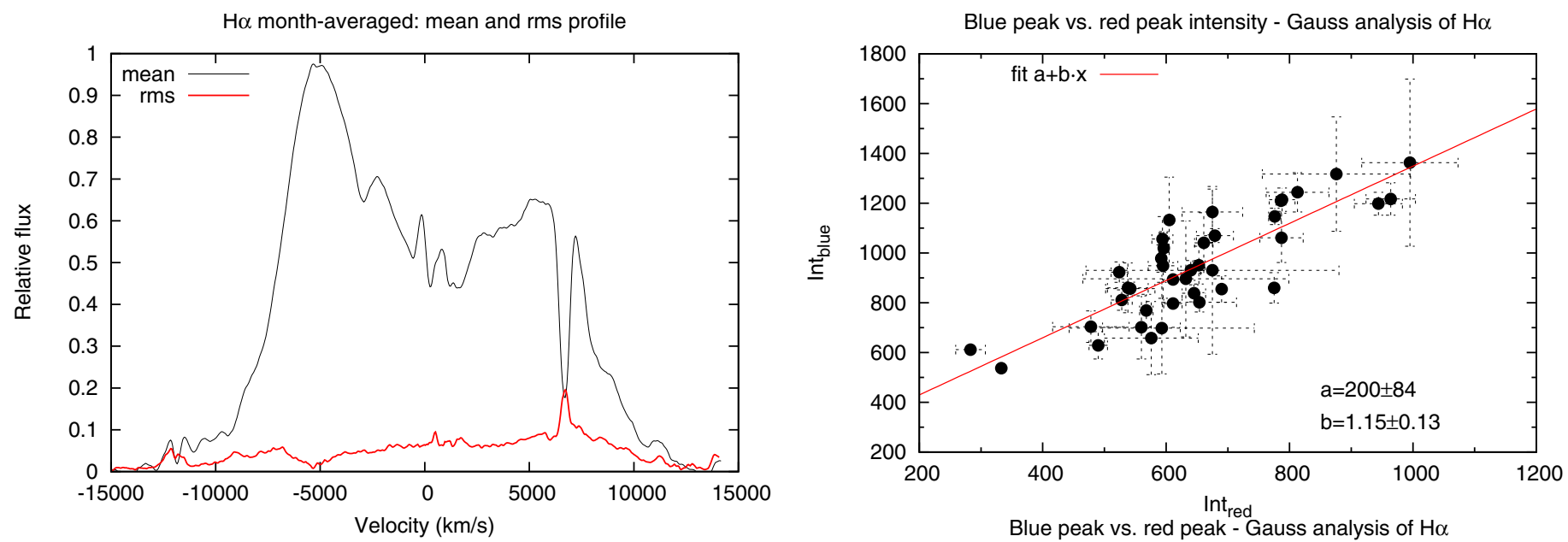

Fig. 8. Mean and rms of the $\mathrm{H} \alpha$ line profile obtained from the monthlyaveraged profiles normalized to the intensity of the blue peak.
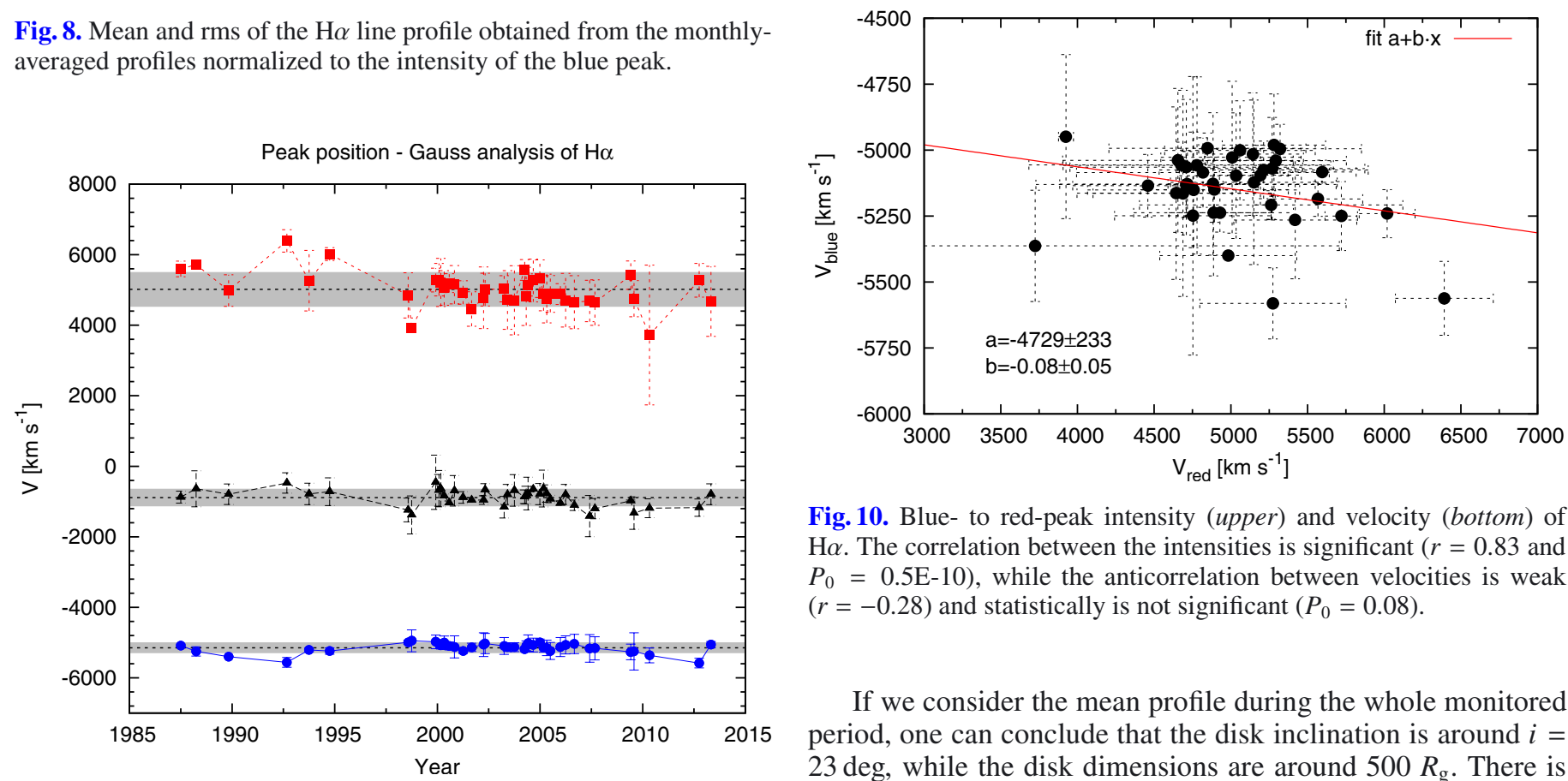

Fig. 10. Blue- to red-peak intensity (upper) and velocity (bottom) of $\mathrm{H} \alpha$. The correlation between the intensities is significant $(r=0.83$ and $\left.P_{0}=0.5 \mathrm{E}-10\right)$, while the anticorrelation between velocities is weak $(r=-0.28)$ and statistically is not significant $\left(P_{0}=0.08\right)$.

If we consider the mean profile during the whole monitored period, one can conclude that the disk inclination is around $i=$ $23 \mathrm{deg}$, while the disk dimensions are around $500 R_{\mathrm{g}}$. There is agreement between the disk parameters from our fit of the mean $\mathrm{H} \alpha$ profile and that given in Gezari et al. (2007), apart from the disk inclination. However, by varying other parameters we were able to obtain $i \sim 30 \mathrm{deg}$, but in all fittings the disk dimensions seem to be very compact (about several $100 \mathrm{~s}$ gravitational radii). In Fig. 13, we plotted both models together with the normalized mean $\mathrm{H} \alpha$ profile.

We conclude the line shape (i.e., double-peaked profiles) can be mainly fitted with a simple relativistic disk model, however, to fit the same line from different periods, one should change the disk parameters, such as inclination or emissivity. This, in principle is not expected, but we should point out that the obtained parameters from the fit are strongly dependent on the positions and intensities of the blue and red peaks, and as we noted above, there are relatively big uncertainties in the red-peak properties.

\section{Discussion}

Double-peaked broad emission lines signature expected from the disk-like BLR are observed in the spectrum of Arp 102B (Chen \& Halpern 1989; Eracleous \& Halpern 1994; Sergeev et al. 2000; Gezari et al. 2007; Shapovalova et al. 2013). The double-peaked lines are presented in a small fraction of AGNs

Fig. 9. Variability of the peak velocity obtained from the Gaussian fitting (red, central, blue) of $\mathrm{H} \alpha$ (upper) and $\mathrm{H} \beta$ line (bottom). 

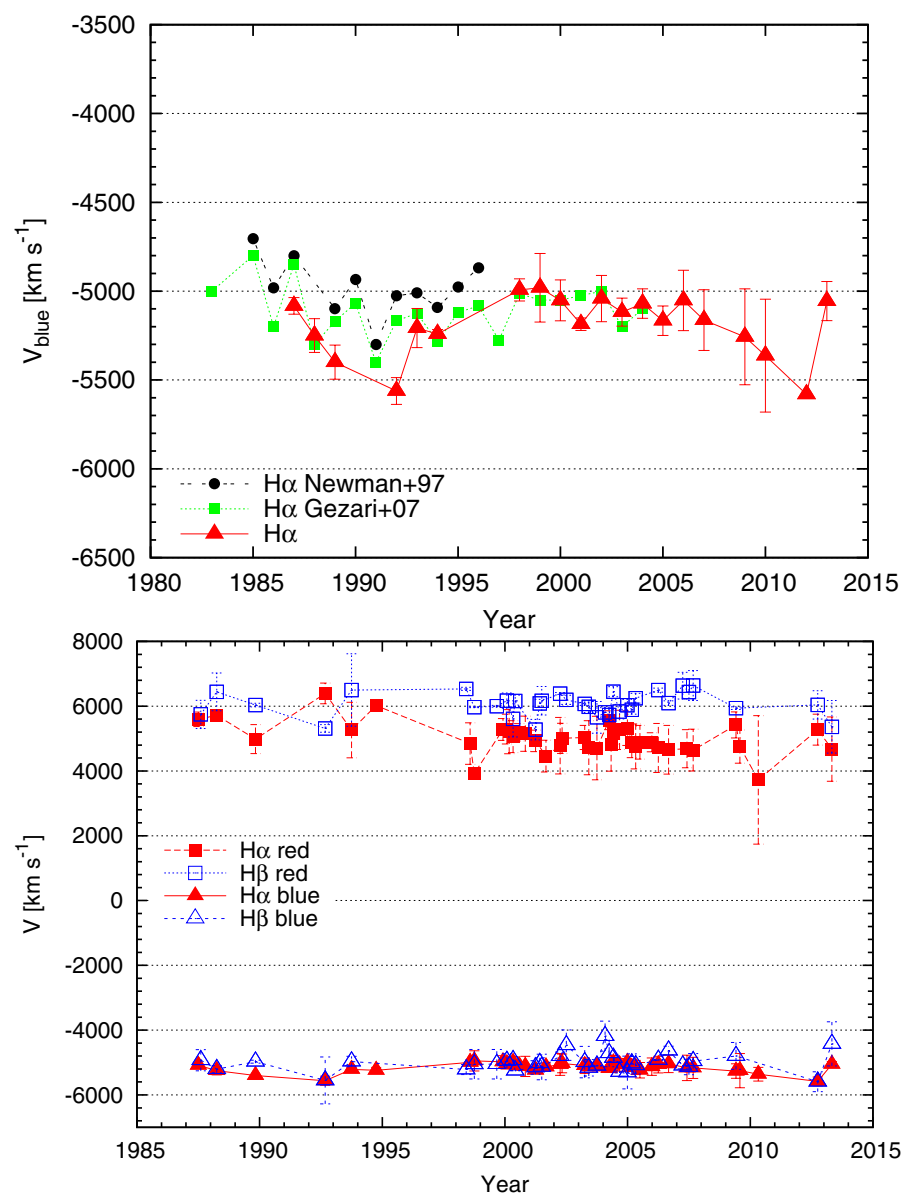

Fig. 11. Upper panel: year-averaged radial velocities of the blue peak. Full squares: the $\mathrm{H} \alpha$ data from Gezari et al. (2007) year-averaged to match our data; full circles: the $\mathrm{H} \alpha$ given in Newman et al. (1997) year-averaged to match our data; full triangles: our data for $\mathrm{H} \alpha$. Bottom panel: month-averaged radial velocities of the blue- and redpeak positions for $\mathrm{H} \alpha$ and $\mathrm{H} \beta$.

(see Strateva et al. 2003). The double-peaked line profiles have been explained with a number of different models: a) binary black holes (see, e.g., Gaskell 1983); b) bipolar outflows (see, e.g., Zheng et al. 1991); c) anisotropically illuminated spherical distribution of optically thick clouds (Goad \& Wanders 1996); d) circular or elliptical accretion disk (see, e.g., Chen et al. 1989; Chen \& Halpern 1989; Eracleous et al. 1995, 1996), and e) other more sophisticated models like precessing elliptical disk or a circular disk with a long-lived, single-armed spiral or warp (see, e.g., Gezari et al. 2007; Jovanović et al. 2010).

Here we can exclude the binary black hole (see, e.g., Gaskell 1983) model, since there is no significant change in the position of the line peaks. Additionally, the model of an anisotropically illuminated spherical distribution of optically thick clouds (Goad \& Wanders 1996) does not explain the big distance between the line peaks (more than $10000 \mathrm{~km} \mathrm{~s}^{-1}$ ), therefore here we will consider the disk-like geometry of the BLR and the possibility of outflows in the BLR.

\subsection{The BLR disk emission: pros and cons}

As we noted above, the disk emission has usually been assumed to model double-peaked profiles of Arp 102B, however, some observational facts from monitoring of the broad lines
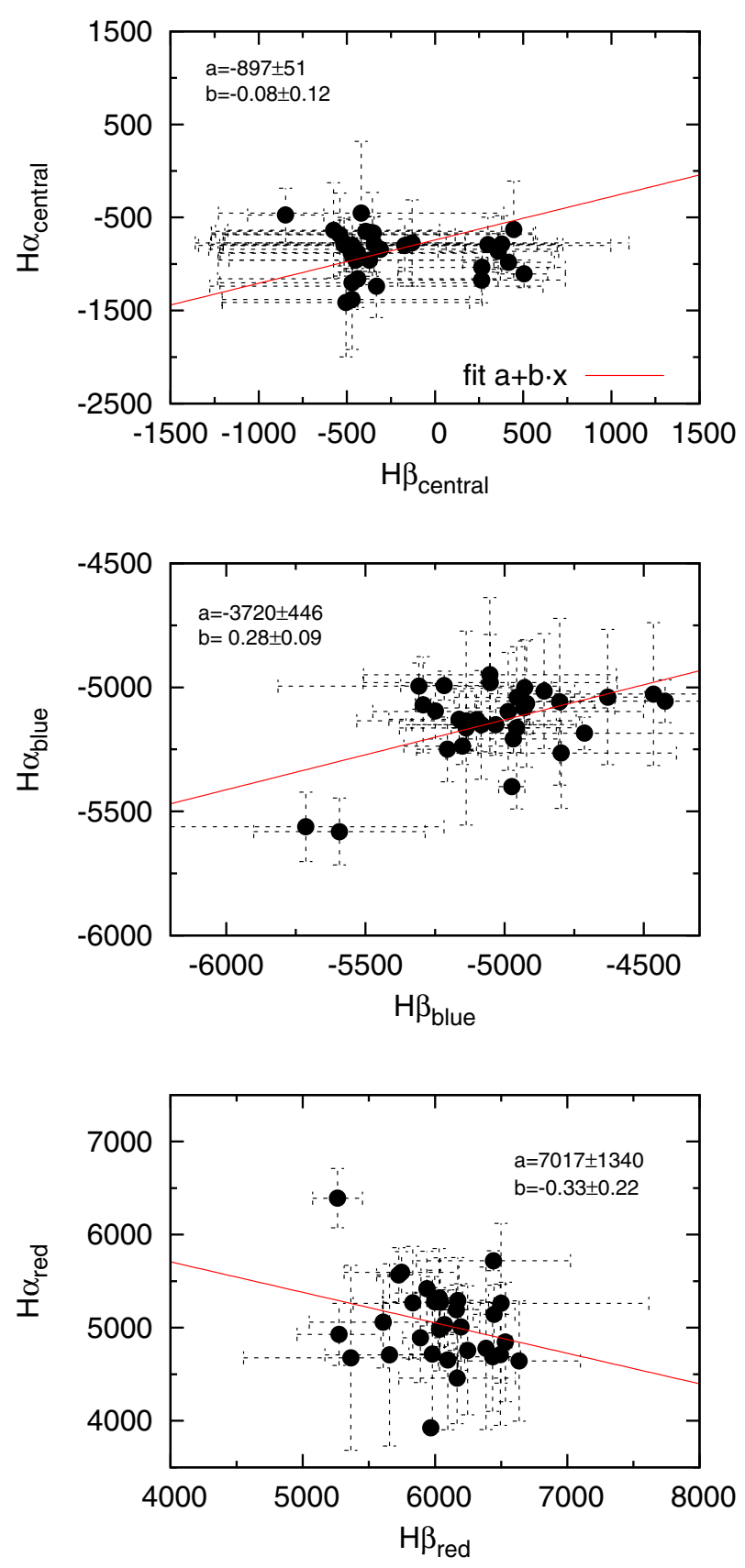

Fig. 12. $\mathrm{H} \alpha$ vs. $\mathrm{H} \beta$ peak velocity for the central (upper), blue (middle), and red (bottom) peak from the month-averaged profiles. The dashed line represents the expected function for an equal peak position in both lines, while the solid line represents the best fit of the measured data. The correlation coefficients are: $r=-0.12\left(P_{0}=0.5\right)$ for the central, $r=0.51\left(P_{0}=0.3 \mathrm{E}-02\right)$ for the blue peak, and $r=-0.26\left(P_{0}=0.15\right)$ for the red peak. The parameters of the best fit are given on the plots.

contradicts the disk model. Recall here some of the observational facts (see Miller \& Peterson 1990; Gezari et al. 2004, 2007):

- Flares in the broad line flux that can also be seen in our observations (see Paper I), but this can be expected if there are some transient processes in the disk (see, e.g., Jovanović et al. 2010).

- Systematic variations in FWQM reported in Gezari et al. (2004), which also may be explained in the disk structure variation. 


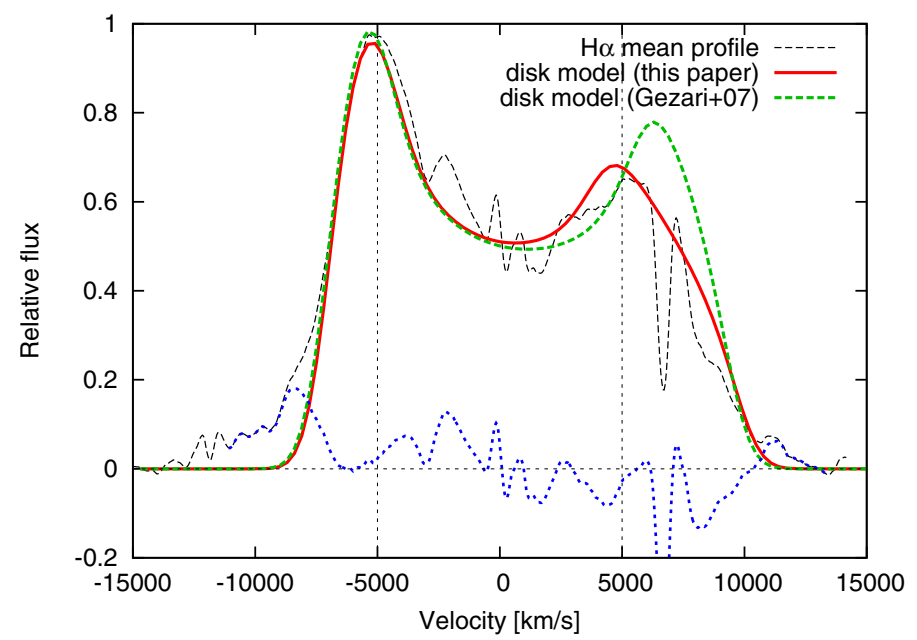

Fig. 13. Mean normalized $\mathrm{H} \alpha$ line profile fitted with the disk-model: solid line - our assumption (see text) and thick dashed line - parameters taken from Gezari et al. (2007). The residual at the bottom corresponds to the difference between the disk model from this paper and mean profile.

- Periodic oscillation of the red-to-blue wing flux ratio observed by Newman et al. (1997) and Shapovalova et al. (2013) can also be caused by rotating structure in the disk.

- Intensity of the red peak is sometimes higher than the blue peak (see Figs. 3 and 4), which can also be explained by perturbations in the disk structure (see, e.g., Jovanović et al. 2010; Popović et al. 2011) as well as by a model of precessing elliptical disk (Gezari et al. 2007).

As one can see, some observed facts mentioned above can be explained by a more complex and nonstable configuration of the accretion disk, i.e., that emissivity enhancements such as transient shocks induced by tidal perturbations could be present, and that the change in line profile can be reproduced by changing the inner and outer radii of the line-emitting portion of the disk.

However, there are some other observational facts that are in disagreement with the disk model hypothesis:

a) A lack of (or slightly anti-) correlations of the blue-to-red intensity ratio with FWQM, as shown in Fig. 14. In principle, one can expect that in the relativistic disk the ratio between the blue and red peak should increase with increasing of the FWQM of the line, since there should be no change in the inclination. The intensive blue peak indicates that the inner radius is closer to the central black hole. This should result in a more extensive (and less intensive) red part, contributing to the broader line measured at FWQM. One would expect the observed tendency in $I_{\text {blue }} / I_{\text {red }}$ Vs. FWQM should be correlated, but it seems they are anticorrelated (see Fig. 14). However, Lewis et al. (2010) demonstrated that in the case of a model of the non-axisymmetric disk, in some cases the red-to-blue peak ratio increases as the width of the profile increases. Also, note here that some indication of such anticorrelation in other double peaked AGNs can be seen in Lewis et al. (2010). They showed plots of red-to-blue peak ratio as a function of time and from a quick inspection one can see a few cases where the changes do not follow the expectation that the FWQM is decreasing as the red-to-blue peak ratio increases (or the blue-to-red peak ratio decreases).

b) The distance between the position of the red and blue peak is different for the $\mathrm{H} \alpha\left(\sim 10500 \mathrm{~km} \mathrm{~s}^{-1}\right)$ and $\mathrm{H} \beta$
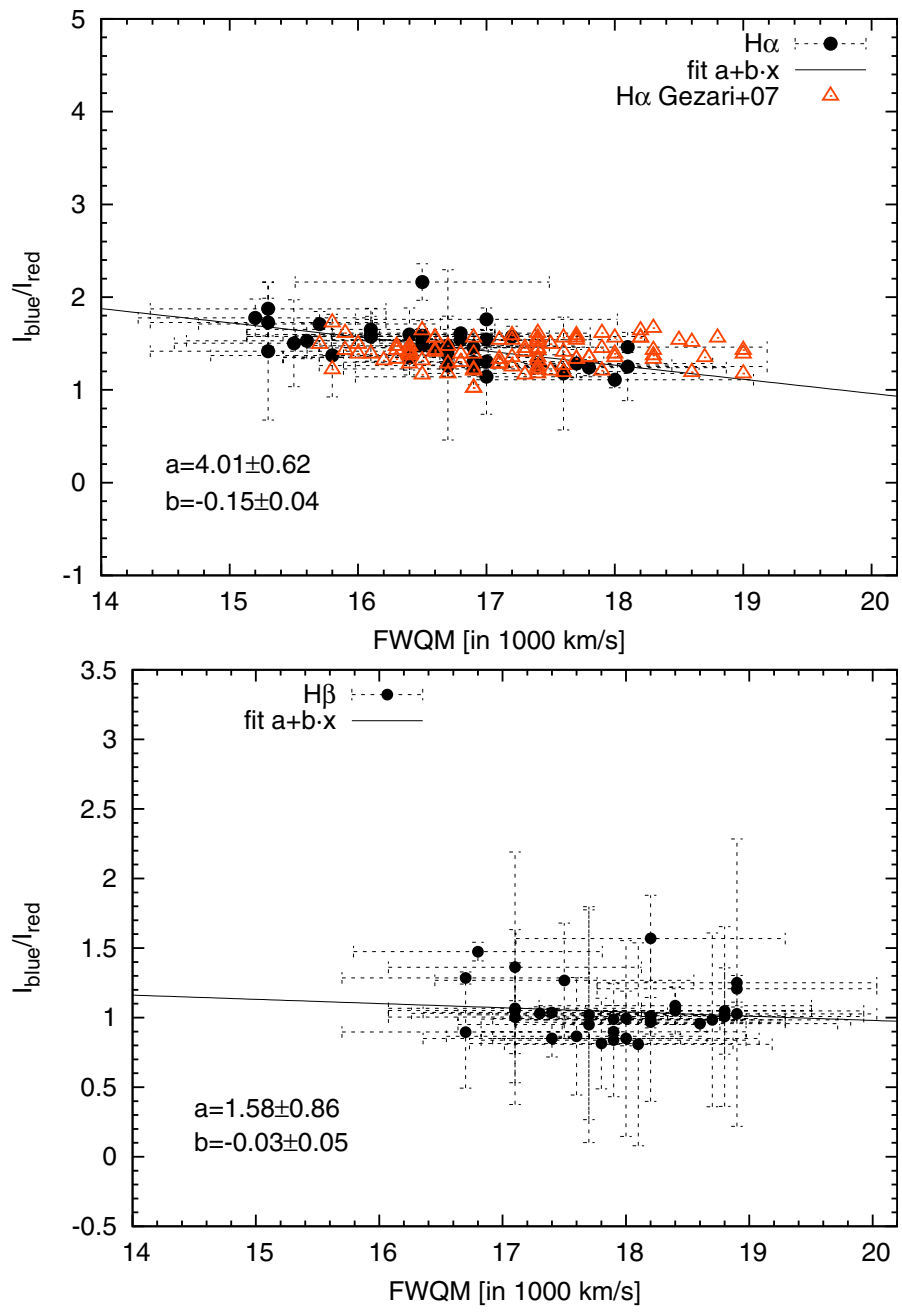

Fig. 14. Blue-to-red peak intensity ratio as a function of FWQM for $\mathrm{H} \alpha$ (upper) and $\mathrm{H} \beta$ (bottom). The correlation coefficients are $r=-0.55$ $\left(P_{0}=0.2 \mathrm{E}-03\right)$ and $r=-0.14\left(P_{0}=0.4\right)$ for $\mathrm{H} \alpha$ and $\mathrm{H} \beta$ respectively. The best fit parameters are given on the plots. The measurements for the $\mathrm{H} \alpha$ of Gezari et al. (2007) are given on upper plot (open triangles).

$\left(\sim 12000 \mathrm{~km} \mathrm{~s}^{-1}\right)$, i.e., the $\mathrm{H} \beta$ shows larger distance for about $1500 \mathrm{~km} \mathrm{~s}^{-1}$ that in the frame of the disk hypothesis should indicate that the line is closer to the central black hole, and consequently that relativistic effects are more observable in the $\mathrm{H} \beta$ line profile. As we see in Figs. 3 and 4, however, the blue boosted peak is more intensive in $\mathrm{H} \alpha$, for almost the entire monitored period. On the other hand, such huge distances between the peaks indicate a fast rotating disk, that is probably close to the black hole. From the rms profile (see Paper I, Fig. 11) one can see that the change in the line profile is also double-peaked, i.e., there is a change in the broad line profile in red and blue peak in both lines, where the variability in the blue wing is significantly bigger than in the red wing. There is one central peak (more intensive in the $\mathrm{H} \beta$ line) in the rms profile that may be caused by a central component (also see Fig. 11 from Paper I).

c) Additionally, it is confusing that there is no big change in the $\mathrm{H} \alpha$ normalized line profile during this long monitored period (see Figs. 3 and 8), as well as in the displacement of the peaks. If the emission is originating in the disk, the disk emission is very stable during the time, i.e., there is no big change in the disk that affects the emission. On the contrary, 


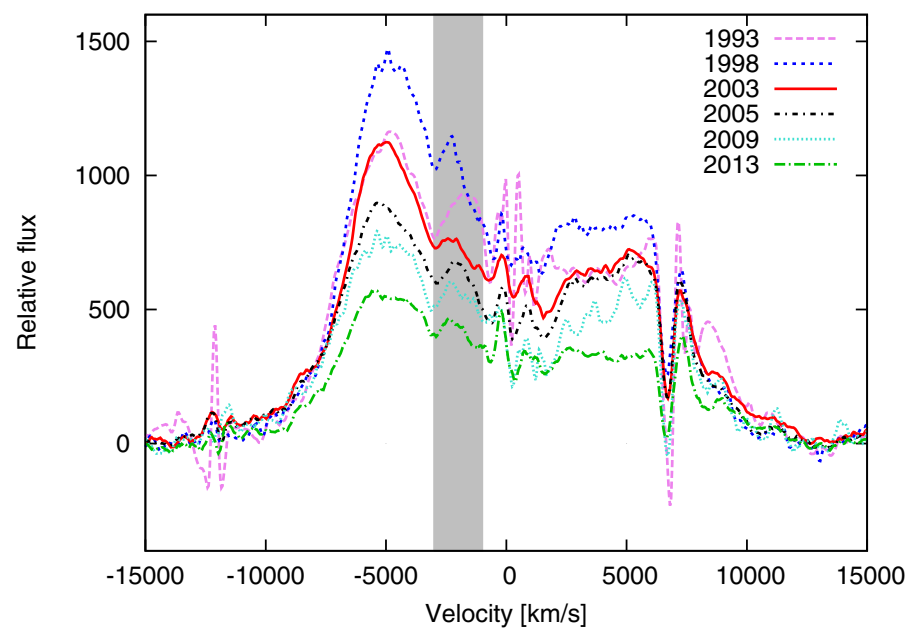

Fig. 15. Bump in the $\mathrm{H} \alpha$ line profile at $\sim 2000 \mathrm{~km} \mathrm{~s}^{-1}$ in years when it is most intensive.

the different peak intensity ratio (e.g., in some periods the red peak of $\mathrm{H} \beta$ is more intensive than the blue peak) indicates that long-living changes in the disk structure should be present (see, e.g., Jovanović et al. 2010; Popović et al. 2011). These changes should affect a compact disk ( $\sim 500$ gravitational radii, that is several light days), and consequently a stronger variability in the broad line profiles is expected.

d) Finally, the spectropolarimetric observations are also inconsistent with the BLR disk model. Spectropolarimetric observations (see Antonucci et al. 1996; Corbett et al. 1998, 2000) did not confirm the disk-like structure of the BLR in Arp 102B. It specially, is interesting that the angle of the jet direction ( $\sim 105$ degrees) corresponds to the polarization angle in the $\mathrm{H} \alpha$ line ( 103 degrees, see Corbett et al. 1998). The $\mathrm{H} \alpha$ polarized shape of Arp 102B indicates a line-emitting biconical outflow surrounded by a cylindrical scattering region (Corbett et al. 1998, 2000). However, there are two facts that prevent us from excluding the disk model. First, that Chen et al. (1997) obtained a qualitative agreement between the disk model, but other possible explanations of the broad-line polarization properties of Arp 102B are possible, e.g., that to the disk emission there is additional outflow emission (see Fig. 15). Second, as it was mentioned in Afanasiev et al. (2014), the role of the interstellar polarization in the polarization properties of the broad lines is very important and after taking it into account, the polarization angle may be changed.

\subsection{Outflow(s) in the Arp 102B BLR}

Recently, Couto et al. (2013) found a two-armed nuclear spiral (see also Fathi et al. 2011) in an extensive field around the nucleus of Arp 102B $\left(2.5 \times 1.7 \mathrm{kpc}^{2}\right)$ and they estimated that the mass outflow rate along one (the east) arm is between $0.26-0.32 M_{\odot} \mathrm{yr}^{-1}$, which is higher than the mass accretion rate. Therefore, one can expect that an outflow is present in the BLR. As shown in Fig. 15, during some periods, there is a bump in the blue part of the $\mathrm{H} \alpha$ line (around $-2500 \mathrm{~km} \mathrm{~s}^{-1}$ ), which may indicate that in addition to the disk emission, there is an outflow in the BLR.

In principle, a biconical outflow model can explain doublepeaked line profiles (as, e.g., Balmer line profiles of 3C 390.3, see Zheng et al. 1991). The blue-shifted and red-shifted peaks are produced by the approaching and receding parts, respectively. Additionally, a model where an accelerating outflow together with an inflow of the emitting gas is dominating in the BLR can explain very complex line profiles (also double-peaked Ilić et al. 2010; León-Tavares et al., in prep.).

Also, spectropolarimetric observations seem to be in favor of the some type of a biconical model (see Corbett et al. 1998, 2000, and corresponding scheme).

As a summary, one can note that the outflow is probably present in the BLR, and seen as a blue bump between the center of the line and blue peak (around $-2500 \mathrm{~km} \mathrm{~s}^{-1}$, see Fig. 15), but it is not clear if the emission from the outflow is dominant in the broad line profiles. Also, one possible problem is relatively small changes in the broad line profiles, which probably should be highre in the case of outflows.

\section{Conclusion}

We analyzed the variability in the broad line properties in a long period of 26 years of Arp 102B, an AGN with prominent doublepeaked broad line profiles. We investigated the broad line profile variations during this period and from our investigations we outline the following conclusions:

a) The broad line profiles have not been significantly changed in a period of 26 years in the sense that during the monitored time, the shift of peaks stays almost unchanged and there are flare-like changes in the line intensity, and some time in the ratio of the blue-to-red peak intensity. This is unusual if there is an emission of a relatively compact accretion disk (dimension smaller than $1000 \mathrm{Rg}$ ), since changes in the intensity ratio of peaks should result in the line widths as well as in the displacement of the peak velocities. Moreover, the intensity ratio of peaks shows a trend of an anticorrelation with the line width, which is opposite from what one can expect from the disk emission. Sometimes, the position of the red peak does not match the accretion disk model predictions (Fig. 6).

b) As an additional conclusion to our earlier one that the variation of fluxes of $\mathrm{H} \beta$ vs. $\mathrm{H} \alpha$ has a small correlation (see Shapovalova et al. 2013), there is practically no correlation between the velocities of the blue and red peaks between the $\mathrm{H} \beta$ and $\mathrm{H} \alpha$ line. Moreover, the line profiles of $\mathrm{H} \beta$ and $\mathrm{H} \alpha$ are different. The $\mathrm{H} \alpha$ line profile has more intensive blue peak, and $\mathrm{H} \beta$ has almost the equivalent intensity of peaks in the most of observations. Also, it is unexpected that the $\mathrm{H} \beta$ is significantly broader $\left(\sim 1500 \mathrm{~km} \mathrm{~s}^{-1}\right)$ than $\mathrm{H} \alpha$, and that the relativistic effect of the blue-boosting is more prominent in the case of $\mathrm{H} \alpha$.

c) An outflow in the BLR seems to be present (from time to time it is seen as the blue bump around $-2500 \mathrm{~km} \mathrm{~s}^{-1}$ ). Although it is not clear how much the outflow can contribute to the broad line emission, it should be taken into account.

At the end, let us conclude that profiles of the broad doublepeaked lines in Arp 102B can be fitted very well with the disk model, but there are several issues in the variation of the broad spectral line properties that are not in agreement with those expected in the variability of the accretion disk structure. The better spectropolarimetric observations/monitoring of Arp 102B are needed to study the polarization properties of the ordinary broad line component in order to clarify the nature of the BLR. 
L. Č. Popović et al.: Spectral monitoring of Arp 102B. II.

Acknowledgements. This work was supported by INTAS (grant N96-0328), RFBR (grants N97-02-17625 N00-02-16272, N03-02-17123, 06-02-16843, N09-02-01136, 12-02-00857a, 12-02-01237a), CONACYT research grants 39560, 54480, and 151494 (Mexico), PROMEP/103.5/08/4722 grant, the Ministry of Education and Science of Republic of Serbia through the project Astrophysical Spectroscopy of Extragalactic Objects (176001), and DFG grant Ko 857/32-1. L.Č.P., W.K. and D.I. are grateful to the Alexander von Humboldt foundation for support in the framework of program "Research Group Linkage". D.I. has been awarded L'Oréal-UNESCO "For Women in Science" National Fellowship for 2014. We would like to thank the anonymous referee for very useful comments.

\section{References}

Afanasiev, V. L., Popović, L. Č., Shapovalova, A. I., Borisov, N. V., \& Ilić, D. 2014, MNRAS, 440, 519

Antonucci, R., Hurt, T., \& Agol, E. 1996, ApJ, 456, L20

Chen, K., \& Halpern, J. 1989, ApJ, 344, 115

Chen, K. Halpern, J. P., \& Filippenko, A. V. 1989, ApJ, 339, 742

Chen, K, Halpern, J. P., \& Titarchuk, L. G. 1997, ApJ, 483, 194

Corbett, E. A., Robinson, A., Axon, D. J., Young, S., \& Hough, J. H. 1998, MNRAS, 296, 721

Corbett, E. A., Robinson, A., Axon, D. J., \& Young, S. 2000, MNRAS, 319, 685

Couto, G. S., Storchi-Bergmann, T., Axon, D. J., et al. 2013, MNRAS, 435, 2982

Dimitrijević, M. S., Popović, L. Č., Kovačević, J., Dačić, M., \& Ilić, D. 2007, MNRAS, 374, 1181

Eracleous, M., \& Halpern, J. P. 1994, ApJS, 90, 1

Eracleous, M., Livio, M., Halpern, J.P., \& Storchi-Bergmann, T. 1995, ApJ, 438, 610
Eracleous, M., Halpern, J. P., \& Livio, M. 1996, ApJ, 459, 89

Eracleous, M., Halpern, J., Gilbert, A., Newman, J. A., \& Filippenko, A. V. 1997, ApJ, 490, 216

Fathi, K., Axon, D. J., Storchi-Bergmann, T., et al. 2011, ApJ, 736, 77

Gaskell, C. M. 1983, Liege International Astrophysical Colloquia, 24, 473

Gezari, S., Halpern, J. P., Eracleous, M., \& Filippenko, A. V. 2004, IAU Symp., 222,95

Gezari, S., Halpern, J. P., \& Eracleous, M. 2007, ApJS, 169, 167

Goad, M., \& Wanders, I. 1996, ApJ, 469, 113

Halpern, J. P., Eracleous, M., Filippenko, A. V., \& Chen, K. 1996, ApJ, 464, 704

Ilić, D., Popović, L. Č., Shapovalova, A. I., et al. 2010, Mem. Soc. Ast. It. Supp., 15,166

Jovanović, P., Popović, L. Ć., Stalevski, M., \& Shapovalova, A. I. 2010, ApJ, 718,168

Lewis, K. T., Eracleous, M., \& Storchi-Bergmann, T. 2010, ApJS, 187, 416

Miller, J. S., \& Peterson, B. M. 1990, ApJ, 361, 98

Newman, J. A., Eracleous, M., Filippenko, A. V., \& Halpern, J. 1997, ApJ, 485, 570

Popović, L. Č.. Mediavilla, E. G., \& Muñoz, J. A. 2001, A\&A, 378, 295

Popović, L. Č., Mediavilla, E., Bon, E., \& Ilić, D. 2004 A\&A, 423, 909

Popović, L. Č., Shapovalova, A. I., Ilić, D., et al. 2011, A\&A, 528, A130

Riffel, R., Rodríguez-Ardila, A., \& Pastoriza, M. G. 2006, A\&A, 457, 61

Shapovalova, A. I., Popović, L. Č., Burenkov, A. N., et al. 2013, A\&A, 559, A10

Sergeev, S. G., Pronik, V. I., \& Sergeeva, E. A. 2000, A\&A, 356, 41

Strateva, I. V., Strauss, M. A., Hao, L., et al. 2003, AJ, 126, 1720

Sulentic, J. W., Zheng, W., Calvani, M., \& Marziani, P. 1990, ApJ, 355, 15

Zheng, W., Veilleux, S., \& Grandi, S. A. 1991, ApJ, 381, 418 


\section{Appendix A: The line fitting procedure and narrow line subtraction}

One of the problems in the measurements of the broad line parameters is the uncertainty in the narrow line subtraction, especially in the red peak of $\mathrm{H} \alpha$ and $\mathrm{H} \beta$, since the narrow lines are right on top of the red peak in both broad lines. Therefore, to find uncertainties in the narrow line subtraction we performed several tests. Additionally, we considered the $B$-band absorption observed near the [SII] doublet in the $\mathrm{H} \alpha$ wavelength range.

\section{A.1. Correction of the B-band absorption near [SII]}

The $B$-band absorption is present in the red wing of the $\mathrm{H} \alpha$ line, near the narrow [SII] doublet (see Fig. A.1). To correct this absorption we used the template spectrum of NGC 4339, which was observed at the same night as Arp 102B with $2.1 \mathrm{~m} \mathrm{GHO}$ telescope with resolution $\sim 8-9 \AA$ on Mar 26, 2003. Then we corrected the $B$-band absorption near the [SII] lines in the $\mathrm{H} \alpha$ spectral region.

In Fig. A.1, we illustrate the correction of the $B$-band absorption. As one can see in Fig. A.1 the absorption is well corrected, but in some spectra the residuals are still present. However, the residuals are weak and cannot affect the narrow line estimates, or the estimates of the red-peak position in $\mathrm{H} \alpha$ (see following section and Figs. A.2). Therefore, we accepted the parameters from the fits where the $B$-band absorption was not corrected.

\section{A.2. Estimation of the narrow line emission}

As we mentioned above, the uncertainties in the estimations of the broad line parameters come mainly from the subtraction of the narrow lines in the $\mathrm{H} \beta$ and $\mathrm{H} \alpha$ spectral range. It is hard to handle the removal of the narrow emission lines in Arp 102B, because the narrow lines are right on top of the red peak in the $\mathrm{H} \alpha$ and $\mathrm{H} \beta$ broad lines. We used two approaches to explore the uncertainty in the narrow line subtractions: i) fitting the narrow lines with Gaussian functions before and after the $B$-band absorption was corrected (as described in Sect. 2.2); and ii) estimation of the broad profile using spline fitting (using DIPSO). In Figs. A. 2 and A.3, we illustrated the subtraction of the narrow lines using these two methods. In Fig. A.2 (fourth panel), we compared the broad $\mathrm{H} \alpha$ line profile obtained after the subtraction of the narrow lines using these two procedures (note that we fitted the spectra before and after the correction for the $B$-band absorption). As shown in Fig. A.3 (bottom panel), the fitted position of the blue and red peaks is also practically the same for $\mathrm{H} \beta$ for both procedures, therefore we used the Gaussian decomposition in our estimates of the narrow line contribution.

\section{A.3. Correction of the fits using the ratio of the narrow lines}

As we mentioned above, some parameters of the narrow lines are fixed in the spectral fit: first of all, the Gaussian velocity dispersions and shift for all narrow lines in the line wavelength range, as well as the ratio of the [OIII]4959,5007, which is fixed to $1: 3$ (Dimitrijević et al. 2007). Also, one cannot expect that the ratio of narrow lines changes over the period of several years. Therefore, one very clear way to test the robustness of the narrow line fittings is to compute the emission line ratios for each combined fit.

The timescale of the observations is long enough that there could be some variation in the narrow emission line ratios,

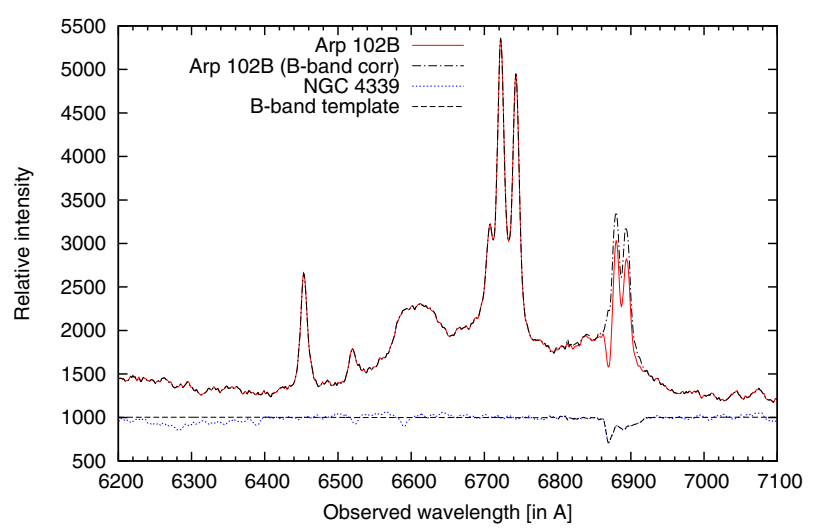

Fig. A.1. Correction of the $\mathrm{H} \alpha$ line on $B$-band absorption. The spectrum of NGC 4399 (shown below) is observed in the same epoch (Mar 26, 2003) as the spectra of Arp 102B.

but certainly over timescales of $\sim 5-10$ years there should not be major changes in the narrow line ratios (except of the random scatter). We calculated the narrow line ratios of $[\mathrm{NII}] / \mathrm{H} \alpha$, $[\mathrm{OI}] 6300 / \mathrm{H} \alpha$, [SII]6715/H $\alpha$, and [OIII]5007/H $\beta$. The line ratios in the $\mathrm{H} \alpha$ and $\mathrm{H} \beta$ wavelength range are presented in Fig. A.4, where dashed lines represent the averaged ratio and shaded regions the $\pm 10 \%$ of the averaged value. As shown in the upper panels of Fig. A.4, there is not any trend in the line ratio variability during the monitored period. Consequently, we corrected those fits for which the narrow line ratios were significantly different from the mean value, i.e., the spectra with a big narrow line ratio difference have been refitted taking the constraint in the fit that the line ratio has to be in the frame of $\pm 15 \%$ of the corresponding mean value. In the bottom panels of Fig. A.4, the line ratios are shown after the correction of a number of spectra.

\section{A.4. The broad line fit and estimates of parameter uncertainties}

To find the broad line parameters of $\mathrm{H} \alpha$ and $\mathrm{H} \beta$ broad lines, we performed a Gaussian analysis, fitting the month-averaged broad line profiles. The broad profile was fitted with three Gaussian functions, corresponding to the blue, central, and red component (Fig. 5). Each Gaussian was described with three free parameters, i.e., in the fit procedure we have nine free parameters: three intensities, widths and shifts of Gaussian functions that describe the blue, central, and red part of the broad line.

Inspection of the obtained parameters from the fit, as well as several additional tests (changing slightly parameters), showed that the central component is often shifted to the blue (especially in $\mathrm{H} \alpha$ ) and that, in some cases, this component has a big (unexpected) change in the shift. Additionally, we found that the position of the central component can significantly affect parameters of the peaks, especially the red peak. Therefore, we repeated the fitting procedure with three broad Gaussian functions, but putting the limits on the shift of the central component to be between $-1000 \mathrm{~km} \mathrm{~s}^{-1}$ and $600 \mathrm{~km} \mathrm{~s}^{-1}$. Using this procedure we obtained an additional set of broad line parameters (parameters of the blue, central, and red Gaussians). Inspection of differences between the parameters obtained from the fits with and without constraint of the central component shift showed that the peak positions are not significantly changed, but the intensities of the red peak, the shift of the central component, and the widths of the components have been significantly changed. 
L. Č. Popović et al.: Spectral monitoring of Arp 102B. II.
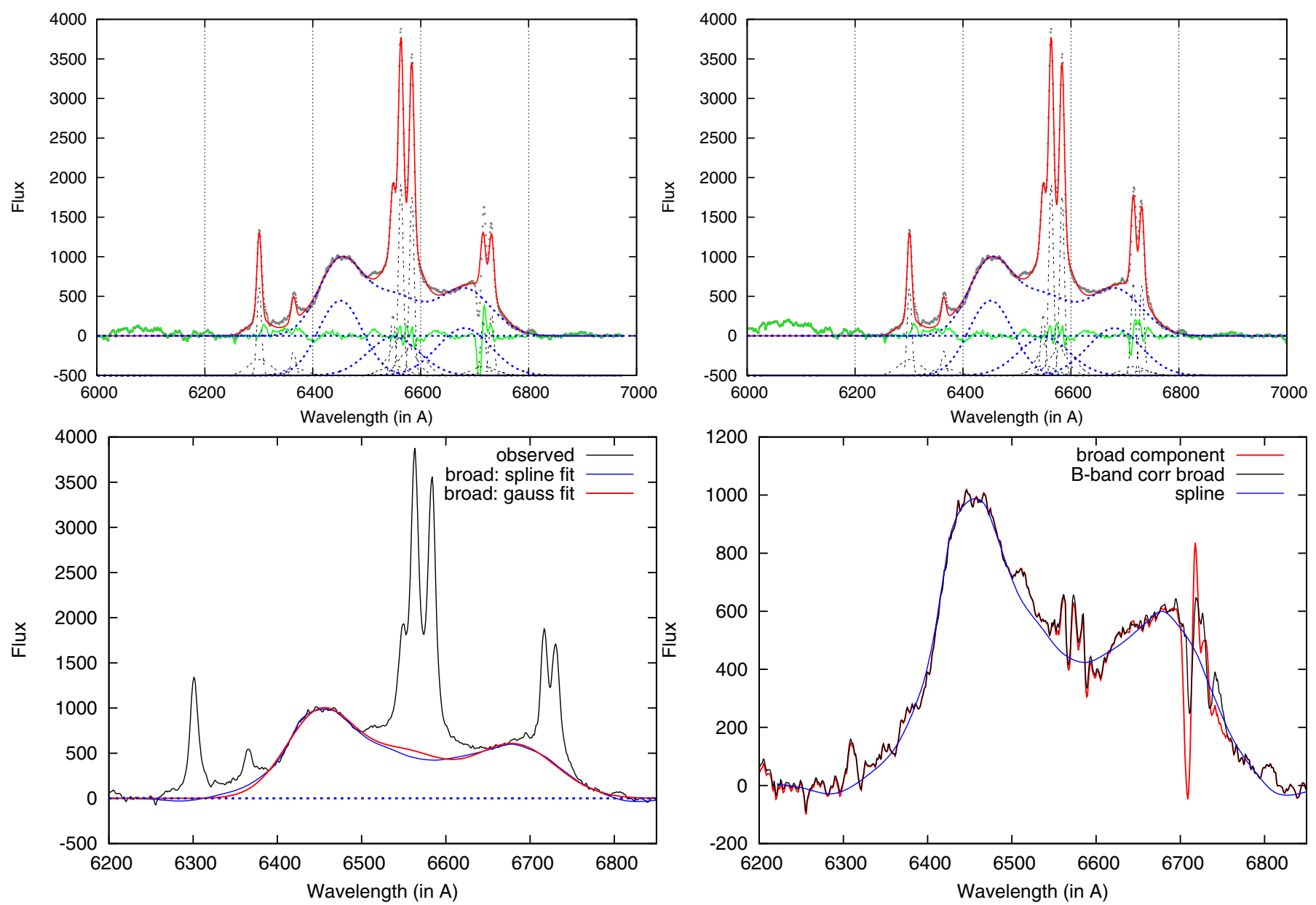

Fig. A.2. Multi-Gaussian fitting of the $\mathrm{H} \alpha$ wavelength range of the spectrum observed on Apr 02, 2003. Upper left: fitting of the spectra where the $B$-band absorption is not corrected. Upper right: the same except for the $B$-band corrected observed spectrum. In these two plots, the same Gaussians parameters (apart for the [SII] lines in which intensities are only increased) have been used. Bottom left: narrow lines removed using the DIPSO spline fitting of the broad component, compared with the 3-Gaussian broad-component fitting. Bottom right: comparison of the H $\alpha$ broad component (after narrow lines subtraction) before and after the $B$-band absorption correction and the broad component obtained using the DIPSO spline fitting. The blue-peak position is the same, a slight difference is seen in the red peak.

We compared the error-bars from the fits with differences between the parameters from these two fitting procedures, and found that the error-bars of parameters are often significantly smaller than the differences between the parameters from both fits. Therefore, we calculated the averaged parameters from the two fits, and accepted uncertainties (error-bars) as the discrepancy between the parameters from the two fits. The averaged broad line parameters and corresponding estimated uncertainties are given in Tables 2 (for $\mathrm{H} \alpha$ ) and 3 (for $\mathrm{H} \beta$ ). 
A\&A 572, A66 (2014)
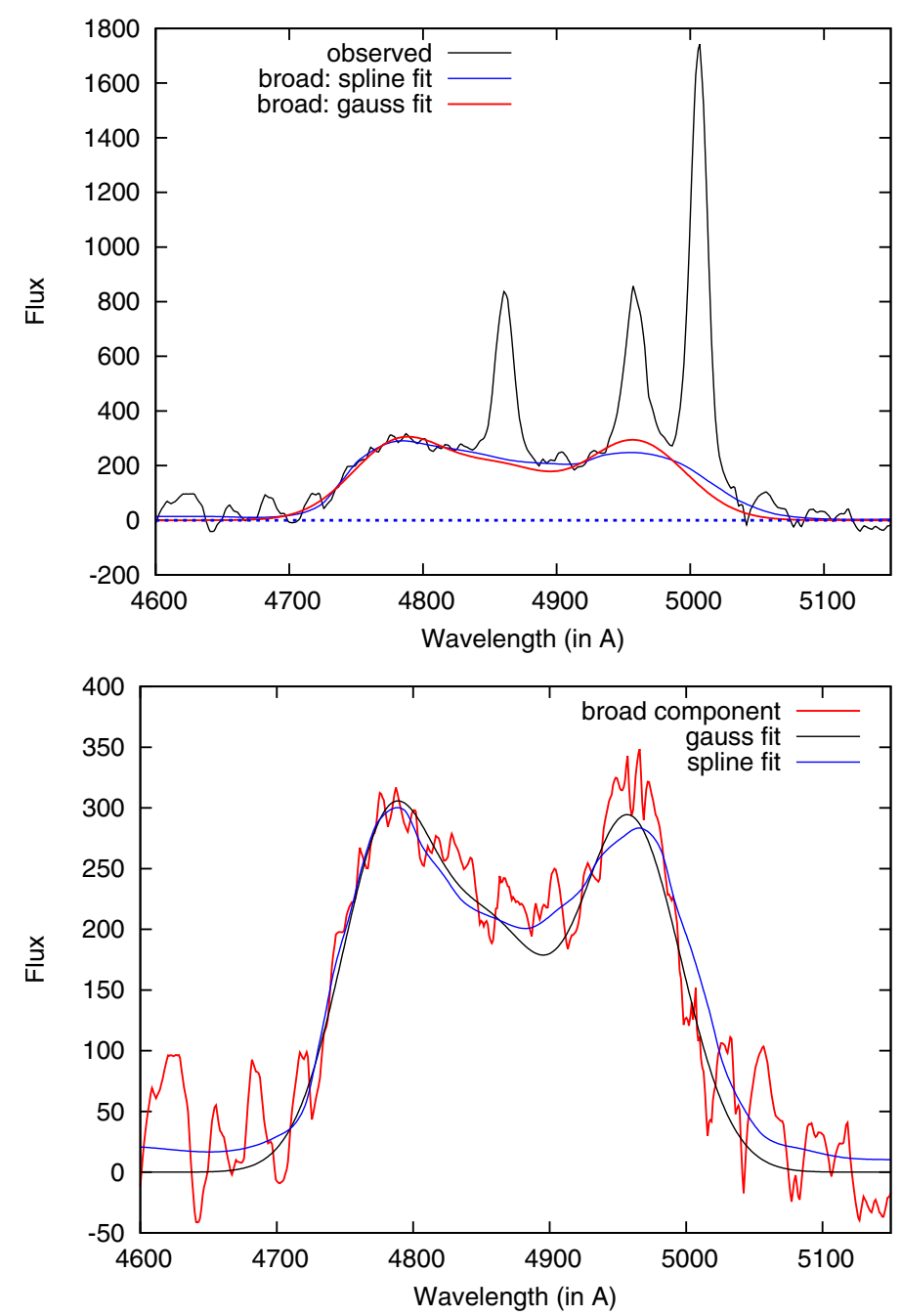

Fig. A.3. Upper panel: narrow lines removed in $\mathrm{H} \beta$ using the DIPSO spline fitting of the broad component, compared with the 3-Gaussian broadcomponent fitting. Bottom panel: comparison of the $\mathrm{H} \beta$ broad component (after narrow lines subtraction) using the 3-Gaussian and DIPSO spline fitting. The blue peak position is the same, a slight difference is seen in the red peak. 
L. Č. Popović et al.: Spectral monitoring of Arp 102B. II.
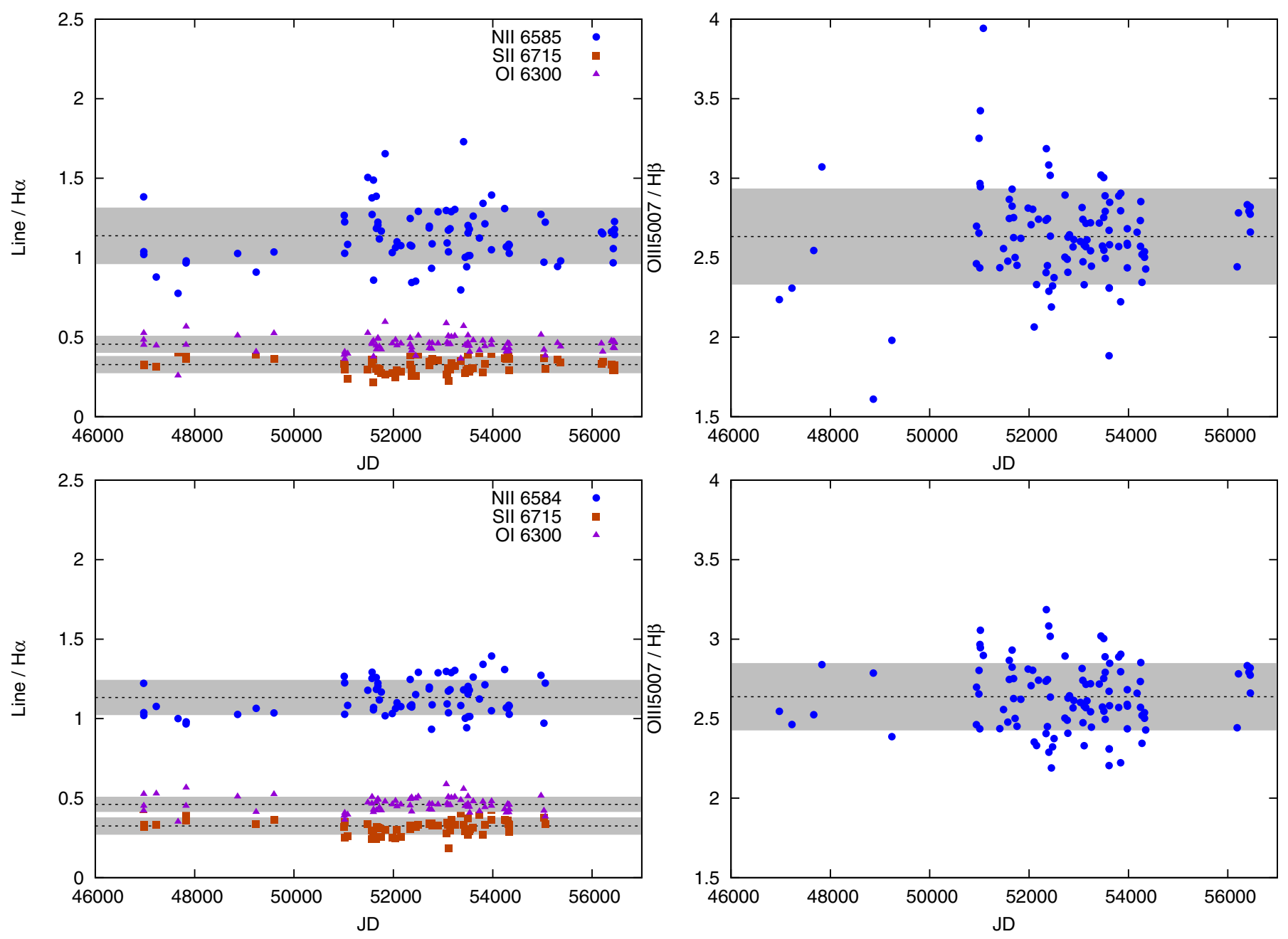

Fig. A.4. Ratio of the narrow emission line (labeled on plots) fluxes in the $\mathrm{H} \alpha$ (left panels) and $\mathrm{H} \beta$ (right panels) wavelength range during the monitored period. The narrow lines fluxes are calculated from the Gaussian fitting parameters as a sum of two components (one narrower fitting the line core and one broader fitting the line wings, see Sect. 2.2) The dashed lines with the shaded regions represent the mean value and the deviation of $10 \%$ from this. In upper panels, we obtained the ratios from the fit without any constraint, while in the bottom panels, the points with large scattering (from upper panels) have been corrected. 